\title{
Local Morrey and Campanato Spaces on Quasimetric Measure Spaces
}

\author{
Krzysztof Stempak ${ }^{1}$ and Xiangxing Tao ${ }^{2}$ \\ ${ }^{1}$ Instytut Matematyki i Informatyki, Politechnika Wrocławska, Wybrzeże Wyspiańskiego 27, 50-370 Wrocław, Poland \\ ${ }^{2}$ Department of Mathematics, Zhejiang University of Science and Technology, Hangzhou, Zhejiang 310023, China \\ Correspondence should be addressed to Xiangxing Tao; xxtao@zust.edu.cn
}

Received 17 February 2014; Accepted 15 April 2014; Published 25 May 2014

Academic Editor: Dachun Yang

Copyright (c) $2014 \mathrm{~K}$. Stempak and X. Tao. This is an open access article distributed under the Creative Commons Attribution License, which permits unrestricted use, distribution, and reproduction in any medium, provided the original work is properly cited.

We define and investigate generalized local Morrey spaces and generalized local Campanato spaces, within a context of a general quasimetric measure space. The locality is manifested here by a restriction to a subfamily of involved balls. The structural properties of these spaces and the maximal operators associated to them are studied. In numerous remarks, we relate the developed theory, mostly in the "global" case, to the cases existing in the literature. We also suggest a coherent theory of generalized Morrey and Campanato spaces on open proper subsets of $\mathbb{R}^{n}$.

\section{Introduction}

A quasimetric on a nonempty set $X$ is a mapping $d: X \times X \rightarrow$ $[0, \infty)$ which satisfies the following conditions:

(i) for every $x, y \in X, d(x, y)=0$ if and only if $x=y$;

(ii) for every $x, y \in X, d(x, y)=d(y, x)$;

(iii) there is a constant $K \geq 1$ such that, for every $x, y, z \in$ $X$,

$$
d(x, z) \leq K(d(x, y)+d(y, z)) .
$$

The pair $(X, d)$ is then called a quasimetric space; if $K=1$, then $d$ is a metric and $(X, d)$ is a metric space.

Given $r>0$ and $x \in X$, let

$$
B(x, r)=\{y \in X: d(x, y)<r\}
$$

be the "quasimetric" ball related to $d$ of radius $r$ and with center $x$. If $(X, d)$ is a quasimetric space, then, $\mathscr{T}_{d}$, the topology in $X$ induced by $d$, is canonically defined by declaring $G \subset X$ to be open, that is, $G \in \mathscr{T}_{d}$, if and only if, for every $x \in G$, there exists $r>0$ such that $B(x, r) \subset G$ (at this point one easily checks directly that the topology axioms are satisfied for such a definition; note, however, that the balls themselves may not be open sets). Observe that this definition is consistent with the definition of metric topology in case when $d$ is a genuine metric. Moreover, the topology $\mathscr{T}_{d}$ is metrizable, see for instance [1] for references.

Two quasimetrics $d$ and $d^{\prime}$ on $X$ are said to be equivalent, if $c^{-1} d^{\prime}(x, y) \leq d(x, y) \leq c d^{\prime}(x, y)$ with some $c \geq 1$ being independent of $x, y \in X$. It is clear that, for equivalent quasimetrics, induced topologies coincide. Moreover, for any $a>0, d^{a}$ is a quasimetric as well and $\mathscr{T}_{d}=\mathscr{T}_{d^{a}}$. A quasimetric $d$ is called a $q$-metric, for $0<q \leq 1$, provided that

$$
d(x, z) \leq\left(d(x, y)^{q}+d(y, z)^{q}\right)^{1 / q}
$$

holds uniformly in $x, y, z \in X$. It is easily checked that a $q$ metric enjoys the open ball property; that is, every ball related to $d$ is an open set in $\left(X, \mathscr{T}_{d}\right)$. It is also known (see [1]) that, given $d$, for $q$ determined by the equality $(2 K)^{q}=2, d_{q}$ defined by

$$
\begin{aligned}
& d_{q}(x, y) \\
& \quad=\inf \left\{\sum_{j=1}^{n} d\left(x_{j-1}, x_{j}\right)^{q}: x=x_{0}, x_{1}, \ldots, x_{n}=y, n \geq 1\right\}
\end{aligned}
$$


is a metric on $X$ which is equivalent to $d^{q}$; more precisely, $d_{q} \leq d^{q} \leq 4 d_{q}$. Consequently, $d_{(q)}:=\left(d_{q}\right)^{1 / q}$ is a $q$-metric equivalent with $d$; more precisely, $d_{(q)} \leq d \leq 4^{1 / q} d_{(q)}$. Thus, every quasimetric admits an equivalent $q$-metric that possesses the open ball property.

In what follows, if $(X, d)$ is a given quasimetric space, then $X$ is considered as a topological space equipped with the (metrizable) topology $\mathscr{T}_{d}$. It may happen that a ball in $X$ is not a Borel set (i.e., it does not belong to the Borel $\sigma$ algebra generated by $\mathscr{T}_{d}$ ), see, for instance, [1] as an example. To avoid such pathological cases, the assumption that all balls are Borel sets must be made. Then, if $X$ is additionally equipped with a Borel measure $\mu$ which is finite on bounded sets and nontrivial in the sense that $\mu(X)>0$, we say that $(X, d, \mu)$ is a quasimetric measure space (we do not assume that $\mu(B)>0$, for every ball $B$ ). In this paper, we additionally assume (similar to the assumption (1.3) made in [2]) that

$$
\text { all balls in } X \text { are open; }
$$

taking into account what was mentioned above, this assumption does not narrow the generality of our considerations.

Let $(X, d, \mu)$ be a quasimetric measure space. Define the function $\rho_{0}: X \rightarrow[0, \infty)$ by setting

$$
\rho_{0}(x)=\inf \{\varepsilon: \mu(B(x, \varepsilon))>0\}, \quad x \in X .
$$

Observe that if $\rho_{0}(x)>0$, for some $x \in X$, then $\mu\left(B\left(x, \rho_{0}(x)\right)\right)=0$; this is a consequence of the continuity property from below of the measure $\mu$. The property " $\mu(B)>$ 0 , for every ball $B$," is equivalent with the statement that $\rho_{0} \equiv$ 0 .

Given a function $\rho: X \rightarrow(0, \infty]$ such that $\rho_{0}(x)<\rho(x)$, for every $x \in X$, let $\mathscr{B}_{\rho}(x)=\mathscr{B}_{\rho, d}(x)$ denote the family of balls (related to $d$ ) centered at $x$ and with radius $r$ satisfying $\rho_{0}(x)<r<\rho(x)$ (clearly balls with different radii but which coincide are identified as sets). Then we set

$$
\mathscr{B}_{\rho}=\mathscr{B}_{\rho, d}=\bigcup_{x \in X} \mathscr{B}_{\rho}(x) .
$$

Thus, $\mathscr{B}_{\rho}$ denotes the family of all $\rho$-local balls in $X$ with positive measure. In case the lower estimate on the radius, $\rho_{0}(x)<r$, is disregarded, we shall write $\widetilde{\mathscr{B}}_{\rho}$ for the resulting family of balls.

By a $\rho$-local integrability of a real or complex-valued function on $X$, we mean its integrability with respect to the family of balls from $\mathscr{B}_{\rho}$; thus, $f \in L_{\text {loc, } \rho}^{1}(X):=L_{\text {loc, } \rho}^{1}(X, d, \mu)$ provided that $\int_{B}|f| d \mu<\infty$, for every ball $B \in \mathscr{B}_{\rho}$ (and thus also for every $B \in \widetilde{\mathscr{B}}_{\rho}$ ). Note that this notion of local integrability does not refer to compactness. Similarly, for $1 \leq$ $p<\infty$, we define $L_{\text {loc }, \rho}^{p}(X)=\left\{f:|f|^{p} \in L_{\text {loc }, \rho}^{1}(X)\right\}$.

If $\rho(x) \neq \infty$, for some $x \in X$, then we will refer to $\rho$ as a locality function and to objects associated to $\rho$ as "local" objects. If $\rho \equiv \infty$ identically, then we shall skip the $\infty$ subscript writing $\widetilde{\mathscr{B}}, L_{\text {loc }}^{1}(X), M_{p, \phi}(X), \mathscr{L}_{p, \phi}(X)$, and so on (thus $\widetilde{\mathscr{B}}$ denotes the family of all balls in $X$ ) and refer to this setting as to the global one. Notice that the proofs of all results stated in the paper contain $\rho=\infty$ as a special case.
Parallel to the main theory, we shall also develop an alternative theory in the framework of closed balls $\bar{B}(x, r)=$ $\{y \in X: d(x, y) \leq r\}$. Note that, in the metric case, $\bar{B}(x, r)$ is indeed a closed set and, in general, if all balls are assumed to be Borel sets, then $\bar{B}(x, r)$ is Borel, too. The definitions of Morrey and Campanato spaces based on closed balls (in fact being closed cubes) in the framework of $\left(\mathbb{R}^{n}, d^{(\infty)}, \mu\right)$ occur in the literature, compare, for instance, [3]. Clearly taking closed balls makes no difference with respect to the theory based on open balls, when $\mu$ has the property that $\mu(\partial B)=0$, for every ball $B$, where $\partial B=\bar{B} \backslash B$; this happens, for instance, when $d \mu(x)=w(x) d x$, where $w \geq 0$ and $d x$ denotes Lebesgue measure on $\mathbb{R}^{n}$. In general, however, the two alternative ways may give different outcomes. Relevant comments indicating coincidences or differences of both theories will be given in several places.

The general notion of local maximal operators was introduced in [4] and some objects associated to them, mostly the BMO spaces, were investigated there in the setting of measure metric spaces. The present paper enhances investigation done in [4] in several directions. First, the broader context of quasimetric measure spaces is considered. Second, the condition $\mu(B)>0$, for every ball $B$, is not assumed. Third, several variants of generalized maximal operators are admitted into our investigation. All this makes the developed theory more flexible in possible applications.

Throughout the paper, we use a standard notation. While writing estimates, we use the notation $S \lesssim T$ to indicate that $S \leq C T$ with a positive constant $C$ independent of significant quantities. We shall write $S \simeq T$ when simultaneously $S \lesssim T$ and $T \lesssim S$; for instance, $d \simeq d^{\prime}$ means the equivalence of quasimetrics $d$ and $d^{\prime}$, and so forth. By $L^{p}(X)=L^{p}(X, \mu)$, $1 \leq p<\infty$, we shall denote the usual Lebesgue $L^{p}$ space on the measure space $(X, \mu)$. Whenever we refer to a ball, we understand that its center and radius have been chosen (in general, these need not be uniquely determined by $B$ as a set). Then, writing $\tau B$, for a given ball $B=B(x, r)$ and $\tau>0$, means that $\tau B=B(x, \tau r)$. For a function $f \in L_{\text {loc, } \rho}^{1}(X)$, its average in a ball $B=B(x, r) \in \mathscr{B}_{\rho}$ will be denoted by

$$
\langle f\rangle_{B}=\frac{1}{\mu(B)} \int_{B} f d \mu
$$

and similarly for any other Borel set $A, 0<\mu(A)<\infty$, and any $f$, whenever the integral makes sense. When the situation is specified to the Euclidean setting of $\mathbb{R}^{n}$, we shall consider either the metric $d^{(2)}$ induced by the norm $\|\cdot\|_{2}$ or $d^{(\infty)}$ induced by $\|\cdot\|_{\infty}$.

\section{Generalized Local Maximal Operators}

By defining and investigating generalized local Morrey and Campanato spaces on quasimetric measure spaces, we adapt the general approach to these spaces presented by Nakai [2] (and follow the notation used there) and extend the concept of locality introduced in [4]. Also, we find it more convenient to work with relevant maximal operators when investigating 
the aforementioned spaces. An interesting concept of localization of Morrey and Campanato spaces on metric measure spaces recently appeared in [5]; this concept is, however, different from our concept. On the other hand, the concept of locality for Morrey and Campanato spaces on metric measure spaces that appeared in the recent paper [6] is consistent with the one we develop; see Remark 15 for further details.

Let $\phi$ be a positive function defined on $\mathscr{B}_{\rho}$. In practice, $\phi$ will be usually defined on $\widetilde{\mathscr{B}}$, the family of all balls in $X$. Then, a tempting alternative way of thinking about $\phi$ is to treat it as a function $\phi: X \times \mathbb{R}_{+} \rightarrow \mathbb{R}_{+}$and then to define $\phi(B)=\phi(x, r)$, for $B=B(x, r)$. There is, however, a pitfall connected with the fact that in general the mapping $X \times \mathbb{R}_{+} \ni(x, r) \mapsto B(x, r) \in \widetilde{\mathscr{B}}$ is not injective. Hence, we assume that $\phi$ possesses the following property:

$$
\phi\left(x_{1}, r_{1}\right)=\phi\left(x_{2}, r_{2}\right) \quad \text { whenever } B\left(x_{1}, r_{1}\right)=B\left(x_{2}, r_{2}\right) \text {. }
$$

(Thus, for instance, when $X$ is bounded, i.e., $\operatorname{diam}(X)=R_{X}<$ $\infty$, the function $\phi$ must obey the following rule: for every $x_{1}, x_{2} \in X$ and $\left.r_{1}, r_{2}>R_{X}, \phi\left(x_{1}, r_{1}\right)=\phi\left(x_{2}, r_{2}\right)\right)$.

Clearly, working with a general $\phi$ cannot lead to fully satisfactory results. Therefore, in what follows, we shall impose some additional mild (and natural) assumptions on $\phi$ in order to develop the theory. Frequently, in such assumptions, $\phi$ and $\mu$ will be interrelated. Of particular interest will be the functions

$$
\phi_{m, \alpha}(B)=\mu(B)^{\alpha}, \quad \phi_{r, \alpha}(B)=r(B)^{\alpha},
$$

where $\alpha \in \mathbb{R}$ and $r(B)$ denotes the radius of $B$ (the $m$ and $r$ stand for measure and radius, resp.). It is necessary to point out here that, for the second function, in fact, we consider a selector $B \mapsto r(B)$ assigning to any $B$, one of its possible radii (clearly this subtlety does not occur when, for instance, $X=\mathbb{R}^{n}$ ). We shall frequently test the constructed theory on these two functions. Finally, let us mention that it may happen that, for a constant $n>0$ (playing the role of the dimension), we have

$$
\mu(B) \simeq r(B)^{n},
$$

uniformly in $B \in \mathscr{B}_{\rho}$. Then,

$$
\phi_{m, \alpha}(B) \simeq \phi_{r, \alpha}(B)^{n}, \quad B \in \mathscr{B}_{\rho} .
$$

Let the system $(X, d, \mu, \rho, \phi)$ be given. In what follows, by an admissible function on $X$, we mean either a Borel measurable complex-valued function (when the complex case is considered) or a Borel measurable function with values in the extended real number system $\overline{\mathbb{R}}=\mathbb{R} \cup\{ \pm \infty\}$ (when the real case is investigated). Given $1 \leq p<\infty$, we define the generalized local fractional maximal operator $M_{p, \phi, \rho}$ acting on any admissible $f$ by

$$
M_{p, \phi, \rho} f(x)=\sup _{x \in B \in \mathscr{B}_{\rho}} \frac{1}{\phi(B)}\left(\frac{1}{\mu(B)} \int_{B}|f|^{p} d \mu\right)^{1 / p}, \quad x \in X,
$$

where the supremum is taken over all the balls from $\mathscr{B}_{\rho}$ which contain $x$, and its centered version by

$$
\begin{aligned}
M_{p, \phi, \rho}^{c} f(x)= & \sup _{\rho_{0}(x)<r<\rho(x)} \frac{1}{\phi(B(x, r))} \\
& \times\left(\frac{1}{\mu(B(x, r))} \int_{B(x, r)}|f|^{p} d \mu\right)^{1 / p}, \quad x \in X .
\end{aligned}
$$

On the other hand, we define the generalized local sharp fractional maximal operator $M_{p, \phi, \rho}^{\#}$ for any admissible $f$ by

$$
\begin{aligned}
M_{p, \phi, \rho}^{\#} f(x)= & \sup _{x \in B \in \mathscr{B}_{\rho}} \inf _{z \in \mathbb{C}} \frac{1}{\phi(B)} \\
& \times\left(\frac{1}{\mu(B)} \int_{B}|f-z|^{p} d \mu\right)^{1 / p}, \quad x \in X,
\end{aligned}
$$

and similarly for its centered version $M_{p, \phi, \rho}^{\#, c}$. (If spaces of real functions are considered, then the infimum is taken over $z \in$ $\mathbb{R}$; the analogous agreement applies in similar places.)

An alternative way of defining the local sharp maximal operator is

$$
\begin{aligned}
\widetilde{M}_{p, \phi, \rho}^{\#} f(x)= & \sup _{x \in B \in \mathscr{B}_{\rho}} \frac{1}{\phi(B)} \\
& \times\left(\frac{1}{\mu(B)} \int_{B}\left|f-\langle f\rangle_{B}\right|^{p} d \mu\right)^{1 / p}, \quad x \in X,
\end{aligned}
$$

but this makes sense only for $f \in L_{\text {loc, } \rho}^{1}(X)$. Similar comment applies to the analogous definition of $\widetilde{M}_{p, \phi, \rho}^{\#, c}$. Clearly,

$$
M_{p, \phi, \rho}^{\#} f(x) \simeq \widetilde{M}_{p, \phi, \rho}^{\#} f(x), \quad M_{p, \phi, \rho}^{\#, c} f(x) \simeq \widetilde{M}_{p, \phi, \rho}^{\#, c} f(x),
$$

uniformly in $f \in L_{\text {loc, } \rho}^{1}(X)$ and $x \in X$. Observe an advantage of using $M_{p, \phi, \rho}^{\#}$ instead of $\widetilde{M}_{p, \phi, \rho}^{\#}: \widetilde{M}_{p, \phi, \rho}^{\#} f$ is defined for $f \in$ $L_{\text {loc, } \rho}^{1}(X)$ only, while $M_{p, \phi, \rho}^{\#} f$ makes sense for much wider class of admissible functions.

For $\phi=1$, that is, when $\phi \equiv 1$, the maximal operators, $M_{1, \mathbf{1}, \rho}, M_{1, \mathbf{1}, \rho}^{\#}$, and $\widetilde{M}_{1, \mathbf{1}, \rho}^{\#}$, and their centered counterparts were defined and investigated in [4] (in the setting of a metric measure space, in addition, satisfying $\mu(B)>0$, for every ball $B)$.

Another property to be immediately noted is

$$
M_{p_{1}, \phi, \rho} f(x) \leq M_{p_{2}, \phi, \rho} f(x), \quad x \in X
$$

that holds, for $1 \leq p_{1}<p_{2}<\infty$, by an application of Hölder's inequality; similar relation is valid for $M_{p, \phi, \rho}^{\#}$ and $\widetilde{M}_{p, \phi, \rho}^{\#}$ and for the centered versions of the three operators. 
Finally, in case of considering maximal operators based on closed balls, we shall use the notations $\bar{M}_{p, \phi, \rho}, \bar{M}_{p, \phi, \rho}^{\#}$, and so forth. To be precise, the definition of $\bar{M}_{p, \phi, \rho}$ is

$$
\bar{M}_{p, \phi, \rho} f(x)=\sup _{x \in \bar{B} \in \overline{\mathscr{B}}_{\rho}} \frac{1}{\phi(\bar{B})}\left(\frac{1}{\mu(\bar{B})} \int_{\bar{B}}|f|^{p} d \mu\right)^{1 / p}, \quad x \in X
$$

where $\overline{\mathscr{B}}_{\rho}$ denotes the family of all closed balls $\bar{B}=\bar{B}(x, r)$ such that $\rho_{0}(x)<r<\rho(x)$ and similarly for other maximal operators considered above. Note that if $\overline{\rho_{0}}(x)=\inf \{\varepsilon$ : $\mu(\bar{B}(x, \varepsilon))>0\}$, then $\overline{\rho_{0}}(x)=\rho_{0}(x)$; this is a consequence of continuity property of the measure $\mu$.

Remark 1. It may be worth mentioning that the following (local) variant of the Hardy-Littlewood maximal operator,

$$
\widetilde{M}_{\kappa, \rho} f(x)=\sup _{x \in B \in \mathscr{B}_{\rho}} \frac{1}{\mu(\kappa B)} \int_{B}|f| d \mu, \quad x \in X,
$$

and its centered version $\widetilde{M}_{\kappa, \rho}^{c}$, where $\kappa>1$ is given, both fall within the scheme presented here: $\widetilde{M}_{\kappa, \rho}$ coincides with $M_{1, \phi_{\kappa}, \rho}$, where $\phi_{\kappa}(B)=\mu(\kappa B) / \mu(B)$. See [7, p. 493], where $\rho \equiv \infty$ and $\kappa=5$, [8, p. 126], where $\rho \equiv \infty, \kappa>1$, and $\widetilde{M}_{\kappa}$ is considered in the setting of $\mathbb{R}^{n}$ and closed cubes, and [9, p. 469], where $\rho \equiv \infty, \kappa=3$, and $\widetilde{M}_{3}^{c}$ is considered in the setting of $\mathbb{R}^{n}$ and open (Euclidean) balls. This variant is an important substitute of the usual Hardy-Littlewood maximal operator (the limiting case of $\kappa=1$ ) and is used frequently in the nondoubling case. Analogous comment concerns yet another variant of the Hardy-Littlewood maximal operator,

$$
\widetilde{M}_{p, \kappa, \rho} f(x):=\left(\widetilde{M}_{\kappa, \rho}\left(|f|^{p}\right)(x)\right)^{1 / p}, \quad x \in X
$$

$1 \leq p<\infty$, (see [9, p. 470], where its centered version is considered for $\rho \equiv \infty$ and $\kappa=3$ ). Also, the local fractional maximal operator

$$
M^{(\alpha)} f(x)=\sup _{x \in B \in \mathscr{B}_{\rho}} \frac{1}{\mu(B)^{1-\alpha / \tau}} \int_{B}|f| d \mu, \quad x \in X,
$$

where $\mu$ is a Borel measure on $X$ satisfying the upper growth condition

$$
\mu(B(x, r)) \lesssim r^{\tau}
$$

for some $0<\tau \leq n$, with $n$ playing the role of a dimension, uniformly in $r>0$, and $x \in X$ (if $X=\mathbb{R}^{n}, \mu$ is Lebesgue measure, and $\tau=n$, then $M^{(\alpha)}$ is the classical fractional maximal operator) is covered by the presented general approach, since $M^{(\alpha)}$ coincides with $M_{1, \phi_{m,-\alpha / \tau}}$. Finally, a mixture of both,

$$
M_{\kappa, p}^{(\alpha)} f(x)=\sup _{x \in B \in \mathscr{B}_{\rho}}\left(\frac{1}{\mu(\kappa B)^{1-p \alpha / \tau}} \int_{B}|f|^{p} d \mu\right)^{1 / p}, \quad x \in X,
$$

considered in $[10]$ in the setting of $\left(\mathbb{R}^{n}, d^{(\infty)}\right)$, coincides with $M_{p, \phi_{\kappa}}$, where

$$
\phi_{\kappa}(B)=\phi_{\kappa, p, \alpha}(B)=\left(\frac{\mu(\kappa B)^{1-p \alpha / \tau}}{\mu(B)}\right)^{1 / p} .
$$

An interesting discussion of mapping properties of (global) fractional maximal operators in Sobolev and Campanato spaces in measure metric spaces equipped with a doubling measure $\mu$, in addition satisfying the lower bound condition $\mu(B(x, r)) \gtrsim r^{\tau}$, is done by Heikkinen et al. in [11]. Investigation of local fractional maximal operators (from the point of view of their smoothing properties) defined in proper subdomains of the Euclidean spaces was given by Heikkinen et al. in [12]. See also comments at the end of Section 3.1.

The following lemma enhances [4, Lemmas 2.1 and 3.1]. By treating the centered case, we have to impose some assumptions on $\rho_{0}, \rho$, and $\phi$. Namely, we assume that $\rho_{0}$ is an upper semicontinuous function (u.s.c. for short), $\rho$ is a lower semicontinuous function (l.s.c. for short), and $\phi$ satisfies

$\forall r>0$ the mapping $X \ni y \longmapsto \phi(y, r)$ is u.s.c.

It may be easily checked that in case $d$ is a genuine metric, $\rho_{0}$ is u.s.c. and $\phi_{m, \alpha}, \alpha \in \mathbb{R}$, satisfies (26).

Lemma 2. For any admissible $f$ and $1 \leq p<\infty$, the functions $M_{p, \phi, \rho} f, M_{p, \phi, \rho}^{c} f, M_{p, \phi, \rho}^{\#} f$, and $M_{p, \phi, \rho}^{\#, c} f$ are l.s.c., hence, Borel measurable, and the same is true for $\widetilde{M}_{p, \phi, \rho}^{\#} f$ and $\widetilde{M}_{p, \phi, \rho}^{\#, c} f$, when $f \in L_{\mathrm{loc}, \rho}^{1}(X)$.

Proof. In the noncentered case no assumption on $\rho_{0}, \rho$, and $\phi$ is required. Indeed, fix $f$, consider the level set $F_{\lambda}=F_{\lambda}(f)=$ $\left\{x \in X: M_{p, \phi, \rho} f(x)>\lambda\right\}$, and take a point $x_{0}$ from this set. This means that there exists a ball $B \in \mathscr{B}_{\rho}$ such that $x_{0} \in B$ and

$$
\frac{1}{\phi(B)}\left(\frac{1}{\mu(B)} \int_{B}|f|^{p} d \mu\right)^{1 / p}>\lambda .
$$

But the same ball $B$, considered for any $y \in B$, also gives $M_{p, \phi, \rho} f(y)>\lambda$; hence, $B \subset F_{\lambda}$, which shows that the level set is open. Exactly the same argument works for the level set $\left\{x \in X: \widetilde{M}_{p, \phi, \rho}^{\#} f(x)>\lambda\right\}$ except for the fact that, now, in (27), $f$ is replaced by $f-\langle f\rangle_{B}$. Finally, consider the level set $F_{\lambda}^{\#}=\left\{x \in X: M_{p, \phi, \rho}^{\#} f(x)>\lambda\right\}$ and take a point $x_{0}$ from this set. There exists a ball $B \in \mathscr{B}_{\rho}$ and $\varepsilon>0$ such that $x_{0} \in B$ and, for every $z \in \mathbb{C}$, we have $(1 / \phi(B))\left(\left\langle|f-z|^{p}\right\rangle_{B}\right)^{1 / p}>\lambda+\varepsilon$. But the same ball $B$ is good enough, for any $y \in B$, in the sense that $M_{p, \phi, \rho}^{\#} f(y)>\lambda$ and, hence, $B \subset F_{\lambda}^{\#}$, which shows that the level set is open.

In the centered case, we use the assumptions imposed on $\rho_{0}, \rho$, and $\phi$. For $M_{p, \phi, \rho}^{c}$, we write the level set $F_{\lambda}^{c}=F_{\lambda}^{c}(f)=$ $\left\{x \in X: M_{p, \phi, \rho}^{c} f(x)>\lambda\right\}$ as a union of open sets

$$
\begin{aligned}
F_{\lambda}^{c}= & \bigcup_{r>0}\left\{y \in X: \frac{1}{\phi(B(y, r))}\left(\left\langle|f|^{p}\right\rangle_{B(y, r)}\right)^{1 / p}>\lambda\right\} \\
& \cap\left\{y \in X: \rho(y)>r>\rho_{0}(y)\right\} .
\end{aligned}
$$


Each intersection on the right hand side is an open set. Indeed,

$$
\begin{aligned}
\left\{y \in X: \rho(y)>r>\rho_{0}(y)\right\}= & \{y \in X: \rho(y)>r\} \\
& \cap\left\{y \in X: \rho_{0}(y)<r\right\}
\end{aligned}
$$

is open, since, by assumption, $\rho$ is l.s.c. and $\rho_{0}$ is u.s.c. On the other hand, for every fixed $r>0$, the function

$$
X \ni y \longmapsto \frac{1}{\phi(B(y, r))}\left(\left\langle|f|^{p}\right\rangle_{B(y, r)}\right)^{1 / p}
$$

is l.s.c. as well. To show this, note that the limit of an increasing sequence of l.s.c. functions is a l.s.c. function, and, hence, it suffices to consider $f=\chi_{A}, \mu(A)<\infty$. But then

$$
X \ni y \longmapsto \frac{1}{\phi(B(y, r))} \frac{\mu(A \cap B(y, r))^{1 / p}}{\mu(B(y, r))}
$$

is l.s.c. as a product of three l.s.c. functions: $X \ni y \mapsto$ $\mu(A \cap B(y, r))^{1 / p}$ is l.s.c. by continuity of $\mu$ from above, $X \ni$ $y \mapsto(1 / \mu(B(y, r)))$ is l.s.c. by continuity of $\mu$ from below, and, finally, $X \ni y \mapsto \phi(B(y, r))^{-1}$ is l.s.c. as well, by the assumption (26) imposed on $\phi$.

Exactly, the same argument works for the level set $\widetilde{F}_{\lambda}^{\#, c}=$ $\left\{x \in X: \widetilde{M}_{p, \phi, \rho}^{\#, c} f(x)>\lambda\right\}$ except for the fact that, now, in relevant places, $f$ has to be replaced by $f-\langle f\rangle_{B}$. Finally, for the level set $F_{\lambda}^{\#, c}=\left\{x \in X: M_{p, \phi, \rho}^{\#, c} f(x)>\lambda\right\}$, an argument similar to that given above combined with that used for $M_{p, \phi, \rho}^{\#}$ does the job.

To relate maximal operators based on closed balls with these based on open balls, we must assume something more on the function $\phi$. Namely, we assume that $\phi$ is defined on the union $\mathscr{B}_{\rho} \cup \overline{\mathscr{B}}_{\rho}$ (rather than on $\overline{\mathscr{B}}_{\rho}$ only) and consider the following continuity condition: for every $y_{0} \in X$ and $\rho_{0}\left(y_{0}\right)<$ $r_{0}<\rho\left(y_{0}\right)$,

$$
\begin{aligned}
& \phi\left(\bar{B}\left(y_{0}, r_{0}\right)\right)=\lim _{r \rightarrow r_{0}^{+}} \phi\left(B\left(y_{0}, r\right)\right), \\
& \phi\left(B\left(y_{0}, r_{0}\right)\right)=\lim _{r \rightarrow r_{0}^{-}} \phi\left(\bar{B}\left(y_{0}, r\right)\right) .
\end{aligned}
$$

Note that $\phi_{m, \alpha}, \alpha \in \mathbb{R}$, satisfies (32) due to the continuity property of measure; in particular, $\phi \equiv 1$ satisfies (32).

We then have the following.

Lemma 3. Assume that (32) holds. Then, for $1 \leq p<\infty$, we have

$$
\begin{aligned}
& \bar{M}_{p, \phi, \rho} f(x)=M_{p, \phi, \rho} f(x), \quad f \in L_{l o c, \rho}^{p}(X), x \in X, \\
& \widetilde{\widetilde{M}}_{p, \phi, \rho}^{\#} f(x)=\widetilde{M}_{p, \phi, \rho}^{\#} f(x), \quad f \in L_{l o c, \rho}^{p}(X), x \in X,
\end{aligned}
$$

and the analogous identities for their centered counterparts. Consequently, for any $f \in L_{l o c, \rho}^{p}(X)$, the functions $\bar{M}_{p, \phi, \rho} f$, $\bar{M}_{p, \phi, \rho}^{c} f, \widetilde{\widetilde{M}}_{p, \phi, \rho}^{\#} f$, and $\overline{\widetilde{M}}_{p, \phi, \rho}^{\#, c} f$ are l.s.c. and, hence, Borel measurable.
Proof. For every $y_{0} \in X$ and $r_{0}>0$, we have

$$
\bar{B}\left(y_{0}, r_{0}\right)=\bigcap_{r>r_{0}} B\left(y_{0}, r\right), \quad B\left(y_{0}, r_{0}\right)=\bigcup_{0<r<r_{0}} \bar{B}\left(y_{0}, r\right) .
$$

To prove $\geq$ in (33), it is sufficient to check that, for any $B_{0}=$ $B\left(y_{0}, r_{0}\right) \in \mathscr{B}_{\rho}$, such that $x_{0} \in B_{0}$, the following holds:

$$
\begin{aligned}
& \frac{1}{\phi\left(B_{0}\right)}\left(\frac{1}{\mu\left(B_{0}\right)} \int_{B_{0}}|f|^{p} d \mu\right)^{1 / p} \\
& \quad \leq \sup _{x \in \bar{B} \in \overline{\mathscr{B}}_{\rho}} \frac{1}{\phi(\bar{B})}\left(\frac{1}{\mu(\bar{B})} \int_{\bar{B}}|f|^{p} d \mu\right)^{1 / p} .
\end{aligned}
$$

Let $r_{n} \rightarrow r_{0}^{-}$and $r_{n}>\rho_{0}\left(y_{0}\right)$. Then, using the second part of (32), continuity of $\mu$ from below, and the monotone convergence theorem gives

$$
\begin{gathered}
\frac{1}{\phi\left(\bar{B}\left(y_{0}, r_{n}\right)\right)}\left(\frac{1}{\mu\left(\bar{B}\left(y_{0}, r_{n}\right)\right)} \int_{\bar{B}\left(y_{0}, r_{n}\right)}|f|^{p} d \mu\right)^{1 / p} \\
\longrightarrow \frac{1}{\phi\left(B_{0}\right)}\left(\frac{1}{\mu\left(B_{0}\right)} \int_{B_{0}}|f|^{p} d \mu\right)^{1 / p} .
\end{gathered}
$$

Similarly, to prove $\leq$ in (33), it suffices to check that, for any $\overline{B_{0}}=\bar{B}\left(y_{0}, r_{0}\right) \in \overline{\mathscr{B}}_{\rho}$, such that $x_{0} \in \overline{B_{0}}$, the following holds:

$$
\begin{aligned}
& \frac{1}{\phi\left(\overline{B_{0}}\right)}\left(\frac{1}{\mu\left(\overline{B_{0}}\right)} \int_{\overline{B_{0}}}|f|^{p} d \mu\right)^{1 / p} \\
& \leq \sup _{x \in B \in \mathscr{B}_{\rho}} \frac{1}{\phi(B)}\left(\frac{1}{\mu(B)} \int_{B}|f|^{p} d \mu\right)^{1 / p} .
\end{aligned}
$$

Let $r_{n} \rightarrow r_{0}^{+}$and $r_{n}<\rho\left(y_{0}\right)$. Then, using the first part of (32), continuity of $\mu$ from above, and the dominated convergence theorem gives

$$
\begin{gathered}
\frac{1}{\phi\left(B\left(y_{0}, r_{n}\right)\right)}\left(\frac{1}{\mu\left(B\left(y_{0}, r_{n}\right)\right)} \int_{B\left(y_{0}, r_{n}\right)}|f|^{p} d \mu\right)^{1 / p} \\
\longrightarrow \frac{1}{\phi\left(\overline{B_{0}}\right)}\left(\frac{1}{\mu\left(\overline{B_{0}}\right)} \int_{\overline{B_{0}}}|f|^{p} d \mu\right)^{1 / p} .
\end{gathered}
$$

The proof of (34) follows the line of the proof of (33) with the additional information that

$$
\begin{aligned}
& \langle f\rangle_{\bar{B}\left(y_{0}, r_{0}\right)}=\lim _{n \rightarrow \infty}\langle f\rangle_{B\left(y_{0}, r_{n}\right)}, \\
& \langle f\rangle_{B\left(y_{0}, r_{0}\right)}=\lim _{n \rightarrow \infty}\langle f\rangle_{\bar{B}\left(y_{0}, r_{n}\right)}
\end{aligned}
$$

(note that $\left.L_{\text {loc, } \rho}^{p}(X) \subset L_{\text {loc }, \rho}^{1}(X)\right)$. Finally, the proofs of the centered versions go analogously.

Given $(X, d)$, let $\kappa_{K}$ be the "dilation constant" appearing in the version of the basic covering theorem for a quasimetric 
space with a constant $K$ in the quasitriangle inequality; see [13, Theorem 1.2]. It is easily seen that $\kappa_{K}=K(3 K+2)$ suffices (so that if $d$ is a metric, then $K=1$ and $\left.\kappa_{K}=5\right) .(X, d)$ is called geometrically doubling provided that there exists $N \in \mathbb{N}$ such that every ball with radius $r$ can be covered by at most $N$ balls of radii $(1 / 2) r$. In the case when $(X, d, \mu)$ is such that $\rho_{0} \equiv 0$, we say (cf. $[4$, p. 243]) that $\mu$ satisfies the $\rho$-local $\tau$-condition, $\tau>1$, provided that

$$
\mu(\tau B) \lesssim \mu(B), \quad B \in \mathscr{B}_{\rho} .
$$

In what follows, when the $\rho$-local $\tau$-condition is invoked, we tacitly assume that $\rho_{0} \equiv 0$.

The following lemma enhances [4, Proposition 2.2].

Proposition 4. Suppose that $\phi$ and $\mu$ satisfy one of the following two assumptions:

(i) $1 \lesssim \phi$ and $\mu$ satisfies the $\rho$-local $\kappa_{K}$-condition;

(ii) $\mu\left(\kappa_{K} B\right) / \mu(B) \lesssim \phi(B)$ uniformly in $B \in \mathscr{B}_{\rho}$, and $(X, d)$ is geometrically doubling.

Then $M_{1, \phi, \rho}$ maps $L^{1}(X, \mu)$ into $L^{1, \infty}(X, \mu)$ boundedly and consequently; $M_{1, \phi, \rho}$ is bounded on $L^{p}(X, \mu)$, for any $1<p<\infty$.

Proof. The assumption $1 \lesssim \phi$ simply guarantees that $M_{1, \phi, \rho} \lesssim M_{1, \mathbf{1}, \rho}$, while the condition $\mu\left(\kappa_{K} B\right) / \mu(B) \lesssim \phi(B)$ implies $M_{1, \phi, \rho} \lesssim \widetilde{M}_{1, \kappa_{K}, \rho}$. To verify the weak type $(1,1)$ of both maximal operators in the latter replacement, note that, for $M_{1, \mathbf{1}, \rho}$, this is simply the conclusion of a version for quasimetric spaces of [4, Proposition 2.2], while, for $\widetilde{M}_{1, \kappa_{K}, \rho}$, the result is essentially included in [7, Proposition 3.5] ( $\kappa_{K}$ replaces 5 and the argument presented in the proof easily adapts to the local setting). Thus, each of the operators $M_{1, \mathbf{1}, \rho}$ and $\widetilde{M}_{1, \kappa_{K}, \rho}$ is bounded on $L^{p}(X, \mu)$ by applying Marcinkiewicz interpolation theorem, and, hence, the claim for $M_{1, \phi, \rho}$ follows.

Remark 5. It is probably worth pointing out that in the setting of $\mathbb{R}^{n}$, closed cubes, and an arbitrary Borel measure $\mu$ on $\mathbb{R}^{n}$ which is finite on bounded sets, the maximal operator $\widetilde{M}_{\mathcal{K}}$ is of weak type $(1,1)$ with respect to $\mu$ and thus is bounded on $L^{p}(\mu)$, for any $\kappa>1$ (since $\widetilde{M}_{\kappa, \rho} \leq \widetilde{M}_{\kappa}$; the same is true for $\left.\widetilde{M}_{\kappa, \rho}\right)$. The details are given in [8, p. 127]. The same is valid for open (Euclidean) balls; see [14, Theorem 1.6]. In [14], Sawano also proved that, for an arbitrary separable locally compact metric space equipped with a Borel measure which is finite on bounded sets (every such a measure is Radon), for every $\kappa \geq 2$, the associated centered maximal operator $\widetilde{M}_{\kappa}^{c}$ is of weak type $(1,1)$ with respect to $\mu$, and the result is sharp with respect to $\kappa$. See also Terasawa [15], where the same result, except for the sharpness, is proved without the assumption on separability of a metric space but with an additional assumption on the involved measure.

\section{Local Morrey and Campanato Spaces}

The generalized local Morrey and Campanato spaces in the setting of the given system $(X, d, \mu, \rho, \phi)$,

$$
\begin{gathered}
L_{p, \phi, \rho}(X):=L_{p, \phi, \rho}(X, d, \mu), \\
\mathscr{L}_{p, \phi, \rho}(X):=\mathscr{L}_{p, \phi, \rho}(X, d, \mu),
\end{gathered}
$$

$1 \leq p<\infty$, are defined by the requirements

$$
\begin{aligned}
\|f\|_{L_{p, \phi, \rho}} & :=\sup _{x \in X} M_{p, \phi, \rho} f(x)<\infty, \\
\|f\|_{\mathscr{L}_{p, \phi, \rho}} & :=\sup _{x \in X} M_{p, \phi, \rho}^{\#} f(x)<\infty,
\end{aligned}
$$

respectively. Note that the identities

$$
\begin{aligned}
& \sup _{x \in X} M_{p, \phi, \rho}^{c} f(x)=\sup _{x \in X} M_{p, \phi, \rho} f(x), \\
& \sup _{x \in X} M_{p, \phi, \rho}^{\#, c} f(x)=\sup _{x \in X} M_{p, \phi, \rho}^{\#} f(x),
\end{aligned}
$$

hold for any admissible $f$. Therefore, using the centered versions of the operators $M_{p, \phi, \rho}$ and $M_{p, \phi, \rho}^{\#}$ in (43) and (44) does not affect the spaces and the norms. Also,

$$
\sup _{x \in X} \widetilde{M}_{p, \phi, \rho}^{\#, c} f(x)=\sup _{x \in X} \widetilde{M}_{p, \phi, \rho}^{\#} f(x),
$$

and, hence, using either $\widetilde{M}_{p, \phi, \rho}^{\#, c}$ or $\widetilde{M}_{p, \phi, \rho}^{\#}$ in place of $M_{p, \phi, \rho}^{\#}$ in (44) does not affect the spaces, and, due to (17), the norms remain equivalent. It is also worth noting that, in the definitions of the spaces $L_{p, \phi, \rho}(X)$ and $\mathscr{L}_{p, \phi, \rho}(X)$, a priori we do not require $f$ to belong to $L_{\text {loc, } \rho}^{p}(X)$ but, a posteriori, indeed $L_{p, \phi, \rho}(X) \subset L_{\text {loc }, \rho}^{p}(X)$ and $\mathscr{L}_{p, \phi, \rho}(X) \subset L_{\text {loc }, \rho}^{p}(X)$.

Other properties to be observed are the inequality

$$
M_{p, \phi, \rho}^{\#} f(x) \leq M_{p, \phi, \rho} f(x), \quad x \in X,
$$

which holds, for any admissible $f$, and gives

$$
\|f\|_{\mathscr{L}_{p, \phi, \rho}} \leq\|f\|_{L_{p, \phi, \rho},} \quad L_{p, \phi, \rho}(X) \subset \mathscr{L}_{p, \phi, \rho}(X),
$$

and the continuous embeddings

$$
L_{p_{1}, \phi, \rho}(X) \subset L_{p_{2}, \phi, \rho}(X), \quad \mathscr{L}_{p_{1}, \phi, \rho}(X) \subset \mathscr{L}_{p_{2}, \phi, \rho}(X),
$$

for $1 \leq p_{1}<p_{2}<\infty$, that follow from (18) and its version for $M_{p, \phi, \rho}^{\#}$.

When $\rho_{0} \equiv 0$ and $d$ is a metric, for $p=1$ and $\phi=$ $\mathbf{1}$, the space $\mathscr{L}_{1, \mathbf{1}, \rho}(X)$ coincides with the local BMO space $\mathrm{BMO}_{\rho}(X)=\mathrm{BMO}_{\rho}(X, d, \mu)$ defined and investigated in [4] in the setting of a metric measure space satisfying $\mu(B)>0$, for every ball $B$.

Since $\|\cdot\|_{\mathscr{L}_{p, \phi, \rho}}$ is merely a seminorm, a genuine norm is generated by considering the quotient space $\mathscr{L}_{p, \phi, \rho}(X) / W_{0, \rho}$, where the subspace $W_{0, \rho}$ is

$$
W_{0, \rho}=\left\{f \in \mathscr{L}_{p, \phi, \rho}(X):\|f\|_{\mathscr{L}_{p, \phi, \rho}}=0\right\} .
$$


Unlikely to the case of $\rho \equiv \infty, W_{0, \rho}$ may be bigger than the space of constant functions. As it was explained in [4, p. 249], $W_{0, \rho}$ coincides with the space of functions which are constant $\mu$-a.e. on each of $\rho$-components of $X$, where $\rho$-components are obtained by means of the equivalence relation $\sim_{\rho}$ and $x \sim{ }_{\rho} y$ provided that there exist balls $\left\{B_{1}, \ldots, B_{m}\right\} \subset \mathscr{B}_{\rho}$ such that $x \in B_{1}, y \in B_{m}$, and $B_{i} \cap B_{i+1} \neq \emptyset, i=1, \ldots, m-1$.

In what follows we shall abuse slightly the language (in fact, we already did it) using in several places the term norm instead of (the proper term) seminorm.

The definition of the generalized local Morrey and Campanato spaces based on closed balls requires using in (43) and (44) the operators $\bar{M}_{p, \phi, \rho}$ and $\bar{M}_{p, \phi, \rho}^{\#}$, respectively. The resulting spaces are then denoted by $\bar{L}_{p, \phi, \rho}(X)$ and $\overline{\mathscr{L}}_{p, \phi, \rho}(X)$, respectively. Lemma 3 immediately leads to Corollary 6.

Corollary 6. Assume that (32) holds. Then, for $1 \leq p<\infty$, we have

$$
\bar{L}_{p, \phi, \rho}(X)=L_{p, \phi, \rho}(X), \quad \overline{\mathscr{L}}_{p, \phi, \rho}(X)=\mathscr{L}_{p, \phi, \rho}(X),
$$

with identity of the corresponding norms in the first case and equivalence of norms in the second case.

Remark 7. Consider the global case; that is, $\rho \equiv \infty$. In the setting of $\mathbb{R}^{n}$ equipped with the Euclidean distance and Lebesgue measure, the classical Morrey and Campanato spaces $L_{p, \lambda}$ and $\mathscr{L}_{p, \lambda}$ (in the notation from [16]) correspond to the choice of $\phi=\phi_{m, \alpha}$ (up to a multiplicative constant), where $1 \leq p<\infty, \alpha=(\lambda / n-1) / p$, and $0 \leq \lambda \leq n+p$, and are explicitely given by

$$
\begin{aligned}
L_{p, \lambda}= & L_{p, \lambda}\left(\mathbb{R}^{n}\right) \\
= & \left\{f:\|f\|_{(p, \lambda)}\right. \\
& \left.:=\sup _{x \in \mathbb{R}^{n}, r>0}\left(\frac{1}{r^{\lambda}} \int_{B(x, r)}|f(y)|^{p} d y\right)^{1 / p}<\infty\right\}, \\
\mathscr{L}_{p, \lambda}= & \mathscr{L}_{p, \lambda}\left(\mathbb{R}^{n}\right) \\
= & \left\{f:\|f\|_{p, \lambda}\right. \\
& :=\sup _{x \in \mathbb{R}^{n}, r>0} \inf _{z \in \mathbb{C}}\left(\frac{1}{r^{\lambda}} \int_{B(x, r)}|f(y)-z|^{p} d y\right)^{1 / p} \\
< & \infty\} .
\end{aligned}
$$

If $\lambda=0$, then clearly $\mathscr{L}_{p, 0} / \mathscr{C} \cong L_{p, 0}=L^{p}\left(\mathbb{R}^{n}\right)$. It is also known (see [16] for references) that, for $0<\lambda<n, \mathscr{L}_{p, \lambda} / \mathscr{C} \cong$ $L_{p, \lambda}$; for $\lambda=n, \mathscr{L}_{p, n}=\operatorname{BMO}\left(\mathbb{R}^{n}\right)$ and $L_{p, n}=L^{\infty}\left(\mathbb{R}^{n}\right)$; and for $n<\lambda \leq n+p, \mathscr{L}_{p, \lambda}=\operatorname{Lip}_{\alpha}\left(\mathbb{R}^{n}\right)$ with $\alpha=(\lambda-n) / p$. Here, $\mathscr{C}=\mathscr{C}\left(\mathbb{R}^{n}\right)$ denotes the space of all constant functions on $\mathbb{R}^{n}$.
Recall that a quasimetric measure space $(X, d, \mu)$ is called a space of homogeneous type provided that $\mu$ is doubling; that is, it satisfies

$$
\mu(B(x, 2 r)) \lesssim \mu(B(x, r))
$$

uniformly in $x \in X$ and $r>0$; clearly, the doubling condition implies that $\rho_{0} \equiv 0$.

In the framework of a space of homogeneous type $(X, d, \mu)$, a systematic treatment of generalized Campanato, Morrey, and Hölder spaces was presented by Nakai [2]. We refer to this paper for a discussion (among other things) of the relations between these spaces. In the nondoubling case, that is, in the setting of $X=\mathbb{R}^{n}$ and a Borel measure $\mu$ that satisfies the growth condition (23), a theory of Morrey spaces was developed by Sawano and Tanaka [3] and Sawano [17]; for details, see Remarks 13 and 14.

Remark 8. Of course it may happen that $L_{p, \phi, \rho}(X, d, \mu)$ is trivial in the sense that it contains only the null function. The triviality of $L_{p, \phi, \rho}(X, d, \mu)$ is equivalent with the statement that, for every nonnull function $f \in L_{\text {loc, } \rho}^{p}(X)$, there exists $x_{0} \in X$ such that $M_{p, \phi, \rho} f\left(x_{0}\right)=\infty$. For instance, if $X=\mathbb{R}^{n}$ with Lebesgue measure, $\rho \equiv \infty, 1 \leq p<\infty$, and $\phi=\phi_{m, \alpha}$, with $\alpha<-1 / p$, then $M_{p, \phi} f\left(x_{0}\right)=\infty$, for every nonnull $f \in L_{\text {loc }}^{p}\left(\mathbb{R}^{n}\right)$ and every $x_{0} \in \mathbb{R}^{n}$ (so that $L_{p, \lambda}\left(\mathbb{R}^{n}\right)=\{0\}$, for every $\lambda<0)$. Similarly, it may happen that $\mathscr{L}_{p, \phi, \rho}(X, d, \mu)$ is trivial in the sense that it consists of functions from $W_{0, \rho}$ only. This time, the triviality of $\mathscr{L}_{p, \phi, \rho}(X, d, \mu)$ is equivalent with the statement that, for every function $f \in L_{\text {loc, } \rho}^{p}(X) \backslash W_{0, \rho}$, there exists $x_{0} \in X$ such that $M_{p, \phi, \rho}^{\#} f\left(x_{0}\right)=\infty$. For instance, if $X=\mathbb{R}^{n}$ with Lebesgue measure, $\rho \equiv \infty, 1 \leq p<\infty$, and $\phi=\phi_{m, \alpha}$, with $\alpha>-1$, then $M_{p, \phi} f\left(x_{0}\right)=\infty$, for every $f \in L_{\text {loc }}^{p}\left(\mathbb{R}^{n}\right) \backslash \mathscr{C}\left(\mathbb{R}^{n}\right)$ and every $x_{0} \in \mathbb{R}^{n}$ (so that $\mathscr{L}_{p, \lambda}\left(\mathbb{R}^{n}\right)=\mathscr{C}\left(\mathbb{R}^{n}\right)$, for every $\lambda>n+p$; in particular, (48) then implies that $L_{p, \lambda}\left(\mathbb{R}^{n}\right)=\{0\}$, for $\left.\lambda>n+p\right)$.

See also [18] for further remarks on triviality of $L_{p, \phi}\left(\mathbb{R}^{n}\right)$ (the global case; $\mathbb{R}^{n}$ equipped with the Euclidean metric $d^{(2)}$ and Lebesgue measure). In the same place, [18], the following interesting observation is made. Let $\phi:(0, \infty) \rightarrow(0, \infty)$ be a function, $\phi(B)=\phi(r)$, for $B=B(x, r), x \in \mathbb{R}^{n}$, and let $1 \leq p<\infty$ be given. If $\bar{\phi}(r)=\inf _{0<t \leq r} \phi(t)>0$, for every $r>0$, then $\bar{\phi}$ is decreasing and $L_{p, \phi}\left(\mathbb{R}^{n}\right)=L_{p, \bar{\phi}}\left(\mathbb{R}^{n}\right)$ with equivalency of norms. Similarly, if inf ${ }_{r \leq t<\infty} \phi(t) t^{n / p}>0$, for every $r>0$, then for $\widetilde{\phi}(r)=r^{-n / p} \inf _{r \leq t<\infty} \phi(t) t^{n / p}, \tilde{\phi}(r) r^{n / p}$ is increasing and $L_{p, \phi}\left(\mathbb{R}^{n}\right)=L_{p, \tilde{\phi}}\left(\mathbb{R}^{n}\right)$ with equivalency of norms.

In the Euclidean setting of $\mathbb{R}^{n}$ with Lebesgue measure, the definition of the classical Morrey and Campanato spaces by using either the Euclidean balls or the Euclidean cubes (with sides parallel to the axes) gives the same outcome. Choosing balls or cubes means using either the metric $d^{(2)}$ or $d^{(\infty)}$. In the general setting, we consider two equivalent quasimetrics on $X$ and possibly different $\rho$ and $\phi$ functions.

The result that follows compares generalized local Morrey and Campanato spaces for the given system $(X, d, \mu, \rho, \phi)$ 
with these of $\left(X, d^{\prime}, \mu, \rho^{\prime}, \phi^{\prime}\right)$ under convenient and, in some sense, natural assumptions.

Proposition 9. Let $1 \leq p<\infty$ and the system $(X, d, \mu, \rho, \phi)$ be given, and suppose that the triple $\left(d^{\prime}, \rho^{\prime}, \phi^{\prime}\right)$ is different from $(d, \rho, \phi)$. Assume also that there exists $n_{0} \in \mathbb{N}$ such that, for any ball $B \in \mathscr{B}_{\rho, d}$, there exists a covering $\left\{B_{1}^{\prime}, \ldots, B_{n_{0}}^{\prime}\right\}$ of $B$ consisting of balls from $\widetilde{\mathscr{B}}_{\rho^{\prime}, d^{\prime}}$ such that

$$
\phi^{\prime}\left(B_{j}^{\prime}\right) \mu\left(B_{j}^{\prime}\right)^{1 / p} \lesssim \phi(B) \mu(B)^{1 / p}, \quad j=1, \ldots, n_{0} .
$$

Then,

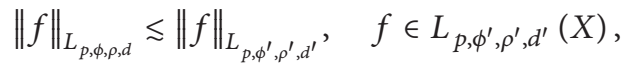

and consequently $L_{p, \phi^{\prime}, \rho^{\prime}, d^{\prime}}(X) \subset L_{p, \phi, \rho, d}(X)$. Similarly, if, for any ball $B \in \mathscr{B}_{\rho, d}$, there exists a ball $B^{\prime} \in \mathscr{B}_{\rho^{\prime}, d^{\prime}}$ such that $B \subset B^{\prime}$ and

$$
\phi^{\prime}\left(B^{\prime}\right) \mu\left(B^{\prime}\right)^{1 / p} \leqslant \phi(B) \mu(B)^{1 / p},
$$

then

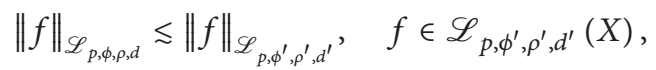

and, hence, $\mathscr{L}_{p, \phi^{\prime}, \rho^{\prime}, d^{\prime}}(X) \subset \mathscr{L}_{p, \phi, \rho, d}(X)$.

Proof. To prove the first claim, take $f \in L_{p, \phi^{\prime}, \rho^{\prime}, d^{\prime}}(X)$ and $B \in$ $\mathscr{B}_{\rho, d}$, and consider a covering of $B, B \subset \cup_{j=1}^{n_{0}} B_{j}^{\prime}$, consisting of balls from $\widetilde{\mathscr{B}}_{\rho^{\prime}, d^{\prime}}$ and satisfying (54). We have

$$
\begin{aligned}
& \frac{1}{\phi(B)}\left(\frac{1}{\mu(B)} \int_{B}|f|^{p} d \mu\right)^{1 / p} \\
& \leq \sum_{j=1}^{n_{0}} \frac{1}{\phi(B) \mu(B)^{1 / p}}\left(\int_{B_{j}^{\prime}}|f|^{p} d \mu\right)^{1 / p} \\
& \leq \sum_{j} \frac{1}{\phi^{\prime}\left(B_{j}^{\prime}\right) \mu\left(B_{j}^{\prime}\right)^{1 / p}}\left(\int_{B_{j}^{\prime}}|f|^{p} d \mu\right)^{1 / p} \\
& \leq n_{0}\|f\|_{L_{p, \phi^{\prime}, \rho^{\prime}, d^{\prime}},}
\end{aligned}
$$

where in the second sum summation goes only over these $j$ 's for which $\mu\left(B_{j}^{\prime}\right)>0$. Taking the supremum over the relevant balls $B$ on the left hand side shows the required estimate and, hence, the inclusion. To prove the second claim, take $f \in \mathscr{L}_{p, \phi^{\prime}, \rho^{\prime}, d^{\prime}}(X)$ and $B \in \mathscr{B}_{\rho, d}$, and consider $B^{\prime}, B \subset B^{\prime}$, satisfying (56). Then

$$
\begin{aligned}
& \inf _{z \in \mathbb{C}} \frac{1}{\phi(B)}\left(\frac{1}{\mu(B)} \int_{B}|f-z|^{p} d \mu\right)^{1 / p} \\
& \quad \leq \inf _{z \in \mathbb{C}} \frac{1}{\phi^{\prime}\left(B^{\prime}\right) \mu\left(B^{\prime}\right)^{1 / p}}\left(\int_{B^{\prime}}|f-z|^{p} d \mu\right)^{1 / p} \\
& \quad \leq\|f\|_{\mathscr{L}_{p, \phi^{\prime}, \rho^{\prime}, d^{\prime}} .}
\end{aligned}
$$

Taking again the supremum over the relevant balls $B$ on the left hand side shows the second required estimate and, hence, the second inclusion.

Corollary 10. Under the assumptions of Proposition 9 and the analogous assumptions but with the roles of $(d, \rho, \phi)$ and $\left(d^{\prime}, \rho^{\prime}, \phi^{\prime}\right)$ switched, we have

$$
\begin{gathered}
L_{p, \phi, \rho, d}(X)=L_{p, \phi^{\prime}, \rho^{\prime}, d^{\prime}}(X), \\
\mathscr{L}_{p, \phi, \rho, d}(X)=\mathscr{L}_{p, \phi^{\prime}, \rho^{\prime}, d^{\prime}}(X),
\end{gathered}
$$

with equivalency of the corresponding norms.

Remark 11. In the case when, in the system $(X, d, \mu, \rho, \phi)$, only $\phi$ is replaced by $\phi^{\prime}$, it may happen that $\phi^{\prime}(B) \lesssim \phi(B)$ uniformly in $B \in \mathscr{B}_{\rho}$. Then, the conclusion of Proposition 9 is obvious but, at the same moment, this is the simplest case of the assumption made in Proposition 9, with $n_{0}=1$ and the covering of $B$ consisting of $\{B\}$.

The following example generalizes the situation of equivalency of theories based on the Euclidean balls or cubes mentioned above.

Example 12. Let $(X, d, \mu)$ be a space of homogeneous type. Assume that $d^{\prime}$ is a quasimetric equivalent with $d$ and $\rho=$ $\rho^{\prime} \equiv \infty$. Given $\alpha \in \mathbb{R}$, let $\phi=\phi_{m, \alpha}^{(d)}$ and $\phi^{\prime}=\phi_{m, \alpha}^{\left(d^{\prime}\right)}$. Then, for $1 \leq p<\infty$ and $\alpha \geq-1 / p$, we have

$$
\begin{aligned}
& M_{p, \phi, d} f(x) \simeq M_{p, \phi^{\prime}, d^{\prime}} f(x), \\
& M_{p, \phi, d}^{\#} f(x) \simeq M_{p, \phi^{\prime}, d^{\prime}}^{\#} f(x),
\end{aligned}
$$

uniformly in $f$ and $x \in X$, and consequently

$$
L_{p, \phi, d}(X)=L_{p, \phi^{\prime}, d^{\prime}}(X), \quad \mathscr{L}_{p, \phi, d}(X)=\mathscr{L}_{p, \phi^{\prime}, d^{\prime}}(X),
$$

with equivalency of the corresponding norms. Indeed, assuming that $c^{-1} d^{\prime} \leq d \leq c d^{\prime}$, for a $c>1$, we have (in what follows $B^{\prime}$ means a ball related to $d^{\prime}$ )

$$
B(x, r) \subset B^{\prime}(x, c r) \subset B\left(x, c^{2} r\right)
$$

and, hence, we take $\left\{B^{\prime}(x, c r)\right\}$ as a covering of $B(x, r) \in \mathscr{B}_{d}$. The doubling property of $\mu$ then implies

$$
\mu\left(B^{\prime}(x, c r)\right) \leq \mu\left(B\left(x, c^{2} r\right)\right) \leq C \mu(B(x, r))
$$

and, therefore, (54) follows with $\phi$ and $\phi^{\prime}$ declared as above. The "dual" estimate follows analogously.

Remark 13. Sawano and Tanaka [3] defined and investigated Morrey spaces in the setting of $\left(\mathbb{R}^{n}, d^{(\infty)}, \mu\right)$, where $\mu$ is a Borel measure on $\mathbb{R}^{n}$ finite on bounded sets (recall that every such measure is automatically a Radon measure) which may be nondoubling. 
For a parameter $k>1$ and $1 \leq p \leq q<\infty$, the Morrey space $\mathscr{M}_{p}^{q}(k, \mu)$ (in the notation of [3] but with the roles of $p$ and $q$ switched) is the space of functions on $\mathbb{R}^{n}$ satisfying

$$
\sup _{\mu(Q)>0} \mu(k Q)^{1 / q-1 / p}\left(\int_{Q}|f|^{p} d \mu\right)^{1 / p}<\infty,
$$

where the supremum is taken over all (closed) cubes with the property $\mu(Q)>0$. The space $\mathscr{M}_{p}^{q}(k, \mu)$ coincides with our space $\bar{L}_{p, \phi}\left(\mathbb{R}^{n}, d^{(\infty)}, \mu\right)$ (i.e., $\left.\rho \equiv \infty\right)$, where

$$
\phi(Q)=\mu(Q)^{-1 / p} \mu(k Q)^{1 / p-1 / q} .
$$

It was proved in [3, Proposition 1.1] (the growth condition (23) did not intervene there) that $\mathscr{M}_{p}^{q}(k, \mu)$ does not depend on the choice of $k>1$. This corresponds to the situation of $d=d^{\prime}=d^{(\infty)}, \rho=\rho^{\prime} \equiv \infty, \phi$ as above and $\phi^{\prime}(Q)=$ $\mu(Q)^{-1 / p} \mu\left(k^{\prime} Q\right)^{1 / p-1 / q}, k^{\prime}>1$, in Corollary 10 since, as it can be easily observed, for $1<k<k^{\prime}<\infty$ say, we have $\phi \leq \phi^{\prime}$ and, on the other hand, the assumption of Proposition 9 is satisfied due to simple geometrical properties of cubes in $\mathbb{R}^{n}$ (see [3, p. 1536] for details).

Remark 14. Sawano [17] defined and investigated the socalled generalized Morrey spaces in the same setting of $\left(\mathbb{R}^{n}, d^{(\infty)}, \mu\right)$ (with closed cubes). For a parameter $k>1$ and a nondecreasing function $\psi:(0, \infty) \rightarrow(0, \infty)$, the space $\mathscr{L}^{p, \psi}(k, \mu)$ was defined as the space of functions on $\mathbb{R}^{n}$ satisfying

$$
\sup _{\mu(Q)>0}\left(\frac{1}{\psi(\mu(k Q))} \int_{Q}|f|^{p} d \mu\right)^{1 / p}<\infty .
$$

The space $\mathscr{L}^{p, \psi}(k, \mu)$ coincides with our space $\bar{L}_{p, \phi}\left(\mathbb{R}^{n}, d^{(\infty)}\right.$, $\mu)$, where

$$
\phi(Q)=\left(\frac{\psi(\mu(k Q))}{\mu(Q)}\right)^{1 / p} .
$$

(Note that, for $\psi(t)=t^{1-p / q}, 1 \leq p \leq q<\infty$, we also have $\left.\mathscr{L}^{p, \psi}(k, \mu)=\mathscr{M}_{p}^{q}(k, \mu)\right)$. It was proved in [1, Proposition 1.2] (again the growth assumption did not intervene there) that $\mathscr{L}^{p, \psi}(k, \mu)$ is independent of $k>1$ with equivalency of norms. This result may also be seen as a consequence of Corollary 10 . Indeed, by taking $\phi^{\prime}(Q)=\left(\psi\left(\mu\left(k^{\prime} Q\right)\right) / \mu(Q)\right)^{1 / p}$ in this corollary, for $1<k<k^{\prime}<\infty$, we have $\phi \leq \phi^{\prime}$. On the other hand, the assumption of Proposition 9 is satisfied by the argument already mentioned in Remark 13 (geometrical properties of cubes in $\mathbb{R}^{n}$ ).

Remark 15. Recently, Liu et al. [6] defined and investigated the local Morrey spaces in the setting of a locally doubling metric measure space $(X, d, \mu)$. The latter means that the measure $\mu$ possesses the doubling and the reverse doubling properties only on a class of admissible balls. This class, $\mathscr{B}_{a}$, is defined with an aid of an admissible function $m: X \rightarrow$ $(0, \infty)$ and a parameter $a \in(0, \infty)$ and agrees with our class $\mathscr{B}_{\rho}$ for the locality function $\rho(x)=a m(x)$ (in [6], an assumption of geometrical nature is imposed on $m$ ). Then, the Morrey-type space $\mathscr{M}_{\mathscr{B}_{a}}^{p, q}(X), 1 \leq q \leq p<\infty$, was defined as the space of functions on $X$ satisfying

$$
\sup _{B \in \mathscr{S}_{a}} \mu(B)^{1 / p}\left(\frac{1}{\mu(B)} \int_{B}|f|^{p} d \mu\right)^{1 / p}<\infty
$$

The investigations in the general setting were next specified in [6] to the important example of the Gauss measure space $\left(\mathbb{R}^{n}, d^{(2)}, \gamma_{n}\right)$, where $\gamma_{n}$ denotes the Gauss measure $d \gamma_{n}(x)=$ $\pi^{-n / 2} \exp \left(-\|x\|_{2}^{2}\right) d x$. The importance of this example lies in the fact that the measure space $\left(\mathbb{R}^{n}, \gamma_{n}\right)$ is the natural environment for the Ornstein-Uhlenbeck operator $-(1 / 2) \Delta+$ $x \cdot \nabla$. In the context of $\left(\mathbb{R}^{n}, d^{(2)}, \gamma_{n}\right)$, the Campanato-type space $\mathscr{E}_{\mathscr{B}_{a}}^{p, q}\left(\gamma_{n}\right)$ was also defined as the space of functions on $X$ satisfying

$$
\|f\|_{L^{1}\left(\gamma_{n}\right)}+\sup _{B \in \mathscr{B}_{a}} \mu(B)^{1 / p}\left(\frac{1}{\mu(B)} \int_{B}\left|f-\langle f\rangle_{B}\right|^{p} d \mu\right)^{1 / p}<\infty
$$

(the additional summand $\|f\|_{L^{1}\left(\gamma_{n}\right)}$ was added due to the specific character of the involved measure space).

Remark 16. In [19, Theorems 4 and 5] an example of Borel measure $\mu$ in $\mathbb{R}^{2}$ was provided ( $\mu$ being absolutely continuous with respect to Lebesgue measure) such that $\mathrm{BMO}^{(2)}\left(\mathbb{R}^{2}, \mu\right)\left(=\mathscr{L}_{1, \mathbf{1}}\left(\mathbb{R}^{2}, d^{(2)}, \mu\right)\right)$ and $\mathrm{BMO}^{(\infty)}\left(\mathbb{R}^{2}, \mu\right)(=$ $\left.\mathscr{L}_{1, \mathbf{1}}\left(\mathbb{R}^{2}, d^{(\infty)}, \mu\right)\right)$ differ.

In the final example of this section, we analyse a specific case that shows that, in general, things may occur unexpected.

Example 17. Take $X=\mathbb{N}, d$ to be the $0-1$ metric on $\mathbb{N}$, and $\mu$ to be the measure on $\mathbb{N}$ such that $\mu(\{n\})=a_{n}$, where $a_{n}>0$ and $\sum a_{n}=1$ (so that $\mu(\mathbb{N})=1$ ). Note that $\mu$ is nondoubling; it is not even locally doubling and if $B$ is a ball, then either $B=\{n\}$, for some $n \in \mathbb{N}$, or $B=\mathbb{N}$, and, hence, $0<\mu(B)<\infty$, for every ball $B$. Then $L_{\text {loc }}^{p}(\mathbb{N})=\ell^{p}(\mathbb{N}, \mu)$, for $1 \leq p<\infty$, and $\rho_{0} \equiv 0$. For simplicity, we now treat the case $\phi=\mathbf{1}$ only.

Consider first $\rho \equiv \infty$. Then, for any $f=(f(j))_{j \in \mathbb{N}} \in$ $\ell^{p}(\mathbb{N}, \mu), M_{p, 1}^{\#} f$ and $M_{p, 1}^{\#, c} f$ are constant functions:

$$
\begin{array}{r}
M_{p, \mathbf{1}}^{\#} f(n)=M_{p, \mathbf{1}}^{\#, c} f(n)=\left(\sum_{j}\left|f(j)-\langle f\rangle_{\mathbb{N}}\right|^{p} a_{j}\right)^{1 / p}, \\
n \in \mathbb{N},
\end{array}
$$

where $\langle f\rangle_{\mathbb{N}}=\sum_{j} f(j) a_{j}$. Hence, $\mathscr{L}_{p, \mathbf{1}}(\mathbb{N})=\ell^{p}(\mathbb{N}, \mu),\|f\|_{\mathscr{L}_{p, 1}}$ $\simeq\left\|f-\langle f\rangle_{\mathbb{N}}\right\|_{\ell^{p}(\mathbb{N}, \mu)}, W_{0}=\mathscr{C}$, and $\mathscr{L}_{p, \mathbf{1}}(\mathbb{N}) / \mathscr{C}$ is identified 
with $\ell^{p}(\mathbb{N}, \mu)$, where $\mathscr{C}=\mathscr{C}(\mathbb{N})$ denotes the space of constant sequences. Similarly, for any $f \in \ell^{P}(\mathbb{N}, \mu)$,

$$
\begin{aligned}
M_{p, \mathbf{1}} f(n) & =M_{p, \mathbf{1}}^{c} f(n) \\
& =\max \left\{|f(n)|,\left(\sum_{j}|f(j)|^{p} a_{j}\right)^{1 / p}\right\}, n \in \mathbb{N},
\end{aligned}
$$

and, hence, $L_{p, \mathbf{1}}(\mathbb{N})=\ell^{\infty}(\mathbb{N}) \cap \ell^{p}(\mathbb{N}, \mu)$ with identity of norms.

Consider now the case of $\rho \equiv 1$. Then $\mathscr{B}_{\rho}$ consists of balls of the form $B=\{n\}, n \in \mathbb{N}, L_{\text {loc }, \rho}^{p}(\mathbb{N})=s(\mathbb{N})$, where $s(\mathbb{N})$ denotes the space of all sequences on $\mathbb{N}$, and $M_{p, 1, \rho}^{\#} f(n)=0$, for any $f \in s(\mathbb{N})$ and $n \in \mathbb{N}$, and, hence, $\mathscr{L}_{p, 1, \rho}(\mathbb{N})=s(\mathbb{N})$ and $\|f\|_{\mathscr{L}_{p, 1, \rho}}=0$, for $f \in s(\mathbb{N})$. In addition, every $\rho$-component is of the form $\{n\}, n \in \mathbb{N}$, and, hence, $W_{0, \rho}=s(\mathbb{N})$. Similarly, for any $f \in s(\mathbb{N})$, we have $M_{p, 1, \rho} f(n)=|f(n)|, n \in \mathbb{N}$, and, hence, $L_{p, \mathbf{1}, \rho}(\mathbb{N})=\ell^{\infty}(\mathbb{N})$ and $\|\cdot\|_{L_{p, 1, \rho}}=\|\cdot\|_{\ell^{\infty}}$.

3.1. Morrey and Campanato Spaces on Open Proper Subsets of $\mathbb{R}^{n}$. In this subsection we suggest a coherent theory of generalized Morrey and Campanato spaces on open proper subsets of $\mathbb{R}^{n}$.

As it was mentioned in [4, p. 259], indecisions accompany choosing a suitable definition of $\mathrm{BMO}(\Omega)$ for a general open proper subset $\Omega$ of $\mathbb{R}^{n}$ equipped with Lebesgue measure. The "right" way seems to be the following: $\mathrm{BMO}(\Omega)$ consists of those functions $f \in L_{\mathrm{loc}}^{1}(\Omega)$ such that

$$
\|f\|_{\mathrm{BMO}(\Omega)}:=\sup _{\bar{B} \subset \Omega} \frac{1}{|\bar{B}|} \int_{\bar{B}}\left|f-\langle f\rangle_{\bar{B}}\right|<\infty,
$$

where the supremum is taken over all closed balls (or closed cubes, if one prefers; then the character $B$ should be replaced by $Q$ ), entirely contained in $\Omega$; see [20]. Throughout this section $|A|$ stands for the Lebesgue measure of $A$, a measurable subset of $\Omega$. Note that such a definition has a local flavor: the locality function entering the scene is

$$
\rho_{\Omega}(x)=d(x, \partial \Omega), \quad x \in \Omega,
$$

where the distance from $x \in \Omega$ to $\partial \Omega$ is given by

$$
d(x, \partial \Omega):=\inf \{d(x, y): y \in \partial \Omega\},
$$

and $d=d^{(2)}$ or $d=d^{(\infty)}$.

Similar indecisions accompany the process of choosing a suitable definition of Morrey and Campanato spaces for a general open proper subset $\Omega \subset \mathbb{R}^{n}$. The spaces $L^{p, \lambda}(\Omega)$, $1 \leq p<\infty$, and $0 \leq \lambda \leq n$, determined by

$$
\|f\|_{L^{p, \lambda}(\Omega)}:=\sup _{x \in \Omega} \sup _{0<r<r_{0}}\left(\frac{1}{r^{\lambda}} \int_{B(x, r) \cap \Omega}|f(y)|^{p} d y\right)^{1 / p}<\infty,
$$

where $r_{0}=\operatorname{diam} \Omega$ (see also [21]), were originally introduced by Morrey [22] (with a restriction to open and bounded subsets). For a definition of $\mathscr{L}^{p, \lambda}(\Omega)$ (nowadays called after Campanato, the Campanato space), also with a restriction to open and bounded subsets, see [23].

An alternative way of defining generalized Morrey and Campanato spaces on open proper (not necessarily bounded) subset $\Omega \subset \mathbb{R}^{n}$ is by using our general approach with the locality function $\rho_{\Omega}$ given above. To fix the attention let us assume, for a moment, that $d=d^{(\infty)}$. Thus, for a given function $\phi: \overline{\mathscr{B}}_{\rho}(\Omega) \rightarrow(0, \infty)$, we define $L_{p, \phi}(\Omega):=$ $\bar{L}_{p, \phi, \rho_{\Omega}}(\Omega)$ and $\mathscr{L}_{p, \phi}(\Omega):=\overline{\mathscr{L}}_{p, \phi, \rho_{\Omega}}(\Omega)$. Explicitely, this means that, for $\rho:=\rho_{\Omega}$,

$$
\begin{aligned}
& L_{p, \phi}(\Omega) \\
& =\left\{f:\|f\|_{L_{p, \phi}(\Omega)}\right. \\
& \left.:=\sup _{\bar{B} \in \overline{\mathscr{B}}_{\rho}(\Omega)} \frac{1}{\phi(\bar{B})}\left(\frac{1}{|\bar{B}|} \int_{\bar{B}}|f(y)|^{p} d y\right)^{1 / p}<\infty\right\}, \\
& \mathscr{L}_{p, \phi}(\Omega) \\
& =\left\{f:\|f\|_{\mathscr{L}_{p, \phi}(\Omega)}\right. \\
& :=\sup _{\bar{B} \in \overline{\mathscr{B}}_{\rho}(\Omega)} \inf _{z \in \mathbb{C}} \frac{1}{\phi(\bar{B})}\left(\frac{1}{|\bar{B}|} \int_{\bar{B}}|f(y)-z|^{p} d y\right)^{1 / p} \\
& <\infty\}
\end{aligned}
$$

by the definition of $\rho, \overline{\mathscr{B}}_{\rho}(\Omega)$ is the family of all closed balls entirely contained in $\Omega$.

Given a parameter $0<k \leq 1$, we now define the locality function $\rho_{k}=\rho_{k, \Omega}$ as

$$
\rho_{k}(x)=k \cdot d(x, \partial \Omega), \quad x \in \Omega,
$$

so that $\rho_{1}=\rho_{\Omega}$. Then, for a function $\phi$ as above, we define $L_{p, \phi, k}(\Omega):=L_{p, \phi, \rho_{k}}(\Omega)$ and $\mathscr{L}_{p, \phi, k}(\Omega):=\mathscr{L}_{p, \phi, \rho_{k}}(\Omega)$. The structure of the above definition of $L_{p, \phi, k}(\Omega)$ and $\mathscr{L}_{p, \phi, k}(\Omega)$ reveals that if $\Omega$ is not connected, then the defined spaces are isometrically isomorphic to the direct sums of the corresponding spaces built on the connected components of $\Omega$ with $\ell^{\infty}$ norm for the direct sum of the given spaces. Indeed, if, for instance, $f \in L_{p, \phi, k}(\Omega), \Omega=\bigcup_{j \in J} \Omega_{j}$ ( is finite or countable), where each $\Omega_{j}$ is a connected component of $\Omega$, and $f_{j}$ denotes the restriction of $f$ to $\Omega_{j}$, then

$$
\|f\|_{L_{p, \phi, k}(\Omega)}=\sup _{x \in \Omega_{x \in \bar{B} \in \overline{\mathscr{B}}_{k}(\Omega)}} \frac{1}{\phi(\bar{B})}\left(\frac{1}{|\bar{B}|} \int_{\bar{B}}|f|^{p}\right)^{1 / p}
$$




$$
\begin{aligned}
& \left.=\sup _{j \in J} \sup _{x \in \Omega_{j x \in \bar{B} \in \overline{\mathscr{B}}_{k}\left(\Omega_{j}\right)}} \sup _{\phi(\bar{B})} \frac{1}{|\bar{B}|} \int_{\bar{B}}|f|^{p}\right)^{1 / p} \\
& =\sup _{j \in J}\left\|f_{j}\right\|_{L_{p, \phi_{j}, k}\left(\Omega_{j}\right)^{\circ}}
\end{aligned}
$$

Thus, without loss of generality, we can assume (and we do this) that $\Omega$ is connected.

The analogous definitions (and comments associated to them) obey $d=d^{(2)}$. To distinguish between the two cases corresponding to the choice of $d^{(2)}$ or $d^{(\infty)}$, when necessary, we shall write $\rho_{k}^{(2)}$ and $\rho_{k}^{(\infty)}, L_{p, \phi, k}^{\mathscr{B}}(\Omega)$ and $L_{p, \phi, k}^{\mathscr{Q}}(\Omega)$, and so forth. Also, the family of balls related to $\rho_{k}^{(2)}$ will be denoted by $\mathscr{B}_{k}^{\Omega}$, while the family of cubes related to $\rho_{k}^{(\infty)}$ will be denoted by $Q_{k}^{\Omega}$.

In what follows, rather than considering a general $\phi$, we limit ourselves to the specific case of $\phi=\phi_{m, \alpha}$. Clearly, $\phi_{m, \alpha}$ satisfies (32) and, hence, distinguishing between open or closed balls (or open or closed cubes) is not necessary. We write $L_{p, \alpha, k}^{\mathcal{Q}}(\Omega)$ in place of $L_{p, \phi_{m, \alpha}, k}^{\mathcal{Q}}(\Omega)$ and similarly in other occurences. Our goal is to prove that the definitions on Morrey and Campanato spaces do not depend on choosing balls or cubes; this is contained in Theorem 20 .

The following propositions partially contain [24, Theorems 3.5 and 3.9] as special cases.

Proposition 18. Let $1 \leq p<\infty$ and $\alpha \geq-1 / p$ be given. The spaces $L_{p, \alpha, k}^{\mathscr{Q}}(\Omega)$ are independent of the choice of the scale parameter $k \in(0,1)$ with equivalence of the corresponding norms. The analogous statement is valid for the spaces $\mathscr{L}_{p, \alpha, k}^{\mathscr{Q}}(\Omega)$.

Proof. Let $0<k<m<1$. We shall prove the inequalities

$$
\begin{gathered}
\|f\|_{L_{p, \alpha, m}^{Q}(\Omega)} \leq C\|f\|_{L_{p, \alpha, k}^{Q}(\Omega)}, \quad f \in L_{p, \alpha, k}^{Q}(\Omega), \\
\|f\|_{\mathscr{L}_{p, \alpha, m}^{Q}(\Omega)} \leq C\|f\|_{\mathscr{L}_{p, \alpha, k}^{Q}(\Omega)}, \quad f \in \mathscr{L}_{p, \alpha, k}^{\mathscr{Q}}(\Omega),
\end{gathered}
$$

which give the inclusions $L_{p, \alpha, k}^{\mathscr{Q}}(\Omega) \subset L_{p, \alpha, m}^{\mathscr{Q}}(\Omega)$ and $\mathscr{L}_{p, \alpha, k}^{\mathbb{Q}}(\Omega) \subset \mathscr{L}_{p, \alpha, m}^{\mathbb{Q}}(\Omega)$. The inequalities opposite to $(80)$ and (81) (with $C=1$ ) are obvious, and thus the opposite inclusions follow.

Consider first the case of (80). There exists $N=N(m$, $k, n)$ such that bisecting any cube $Q \in Q_{m}^{\Omega} N$ times results in obtaining a family $\left\{Q_{j}\right\}$ of $2^{n N}$ congruent subcubes of $Q$ each of them in $Q_{k}^{\Omega}$. Thus

$$
\begin{aligned}
\frac{1}{|Q|^{\alpha}}\left(\frac{1}{|Q|} \int_{Q}|f|^{p}\right)^{1 / p} & \leq \frac{1}{|Q|^{\alpha}} \sum_{j}\left(\frac{1}{|Q|} \int_{Q_{j}}|f|^{p}\right)^{1 / p} \\
& \leq \sum_{j} \frac{1}{\left|Q_{j}\right|^{\alpha}}\left(\frac{1}{\left|Q_{j}\right|} \int_{Q_{j}}|f|^{p}\right)^{1 / p} \\
& \leq 2^{n N}\|f\|_{L_{p, \alpha, k}^{Q}(\Omega)}
\end{aligned}
$$

and the result follows.
Considering (81), we shall apply the procedure similar to that used in the proof of [24, Theorem 3.5]. Take $f \in$ $\mathscr{L}_{p, \alpha, k}^{\mathscr{Q}}(\Omega)$ and $Q \in \mathbb{Q}_{m}^{\Omega} \backslash Q_{k}^{\Omega}$. Then

$$
\inf _{z \in \mathbb{C}} \frac{1}{|Q|^{\alpha}}\left(\frac{1}{|Q|} \int_{Q}|f-z|^{p}\right)^{1 / p}=\frac{1}{|Q|^{\alpha+1 / p}}\|f\|_{L^{p}(Q) / \mathbb{C}^{\prime}}
$$

where $L^{p}(Q) / \mathbb{C}$ is the quotient space and $\|\cdot\|_{L^{p}(\mathrm{Q}) / \mathbb{C}}$ is the quotient norm. Since the dual to $L^{p}(Q) / \mathbb{C}$ is identified with $L_{0}^{p^{\prime}}(Q)$, where $p^{\prime}$ is the exponent conjugate to $p, 1 / p+1 / p^{\prime}=$ 1 , and $L_{0}^{p^{\prime}}(Q)$ denotes the subspace of $L^{p}(Q)$ consisting of functions $f$ with $\int_{Q} f=0$, therefore

$$
\|f\|_{L^{p}(Q) / \mathbb{C}}=\sup \left\{\left|\int_{Q} f \psi\right|: \psi \in L_{0}^{p^{\prime}}(Q),\|\psi\|_{p^{\prime}} \leq 1\right\} .
$$

According to [24, Lemma 3.1], there exist constants $C=$ $C\left(k, m, p^{\prime}, n\right)$ and $N=N(k, m, n)$ such that, for every $Q \in Q_{m}^{\Omega}$ and every function $\psi \in L_{0}^{p^{\prime}}(Q)$, there exist subcubes $\left\{Q_{j} \in\right.$ $\left.Q_{k}^{\Omega}: 1 \leq j \leq N\right\}$ of $Q$ and functions $\left\{\psi_{j} \in L_{0}^{p^{\prime}}(Q): 1 \leq\right.$ $j \leq N\}$ such that $\operatorname{supp}\left(\psi_{j}\right) \subset Q_{j}$ and $\left\|\psi_{j}\right\|_{p^{\prime}} \leq C\|\psi\|_{p^{\prime}}$, for $j=1, \ldots, N$, and $\psi=\sum_{j=1}^{N} \psi_{j}$. Take $\psi \in L_{0}^{p^{\prime}}(Q)$ satisfying $\|\psi\|_{p^{\prime}} \leq 1$, where $Q$ is the cube chosen earlier, and select subcubes $\left\{Q_{j}\right\}_{1}^{N}$ and functions $\left\{\psi_{j}\right\}_{1}^{N}$ with properties as above. Then

$$
\begin{aligned}
\frac{1}{|Q|^{\alpha+1 / p}}\left|\int_{Q} f \psi\right| & =\frac{1}{|Q|^{\alpha+1 / p}}\left|\int_{Q} f\left(\sum_{j=1}^{N} \psi_{j}\right)\right| \\
& \leq \frac{1}{|Q|^{\alpha+1 / p}} \sum_{j=1}^{N}\left|\int_{Q_{j}} f \psi_{j}\right| \\
& =\frac{1}{|Q|^{\alpha+1 / p}} \sum_{j=1}^{N}\left|\int_{Q_{j}}\left(f-f_{Q_{j}}\right) \psi_{j}\right| \\
& \leq C \sum_{j=1}^{N} \frac{1}{\left|Q_{j}\right|^{\alpha+1 / p}}\left(\int_{Q_{j}}\left|f-f_{Q_{j}}\right|^{p}\right)^{1 / p} \\
& \leq C N\|f\|_{\mathscr{L}_{p, \alpha, k}^{Q}(\Omega)}
\end{aligned}
$$

Hence,

$$
\frac{1}{|Q|^{\alpha+1 / p}}\|f\|_{L^{1}(Q) / \mathbb{C}} \leq C N\|f\|_{\mathscr{L}_{p, \alpha, k}^{Q}(\Omega)},
$$

and, consequently, $\|f\|_{\mathscr{L}_{p, \alpha, m}^{Q}(\Omega)} \leq C N\|f\|_{\mathscr{L}_{p, \alpha, k}^{\mathscr{Q}}(\Omega)}$.

Proposition 19. Let $1 \leq p<\infty$ and $\alpha \geq-1 / p$ be given. The spaces $L_{p, \alpha, k}^{\mathscr{B}}(\Omega)$ are independent of the choice of the scale parameter $k \in(0,1)$ with equivalence of the corresponding norms. The analogous statement is valid for the spaces $\mathscr{L}_{p, \alpha, k}^{\mathscr{B}}(\Omega)$. 
Proof. The present proof mimics the one of Proposition 18, since essentially it suffices to replace the character $Q$ by $\mathscr{B}$ and, assuming $0<k<m<1$ are given, to use the following geometrical properties of Euclidean balls. The first one says that there exists $N=N(k, m, n)$ such that every ball $B \in \mathscr{B}_{m}^{\Omega}$ may be covered by a family $\left\{B_{j}\right\}_{1}^{N}$ of balls each of them in $\mathscr{B}_{k}^{\Omega}$ and with radii smaller than that of $B$. The second one (more sophisticated) is contained in [24, Lemma 3.8] and says that there exist constants $C=C\left(k, m, p^{\prime}, n\right)$ and $N=N(k, m, n)$ such that, for every $B \in \mathscr{B}_{m}^{\Omega}$ and every function $\psi \in L_{0}^{p^{\prime}}(B)$, there exist balls $\left\{B_{j} \in \mathscr{B}_{k}^{\Omega}: 1 \leq j \leq N\right\}$ with radii smaller than that of $B$ and functions $\left\{\psi_{j} \in L_{0}^{p^{\prime}}(B): 1 \leq j \leq N\right\}$ such that $\operatorname{supp}\left(\psi_{j}\right) \subset B_{j}$ and $\left\|\psi_{j}\right\|_{p^{\prime}} \leq C\|\psi\|_{p^{\prime}}$, for $j=1, \ldots, N$, and $\psi=\sum_{j=1}^{N} \psi_{j}$. (The fact that radii of $B_{j}$ are smaller than the radius of $B$ is not directly indicated in the statement of [24, Lemma 3.8] but it is implicitly contained in the construction included in the proof of that lemma.)

For the sake of completeness, we include an outline of the proof of the first aforementioned property. We shall use the following simple geometrical fact: given $0 \leq R^{\prime}<R$ and $\varepsilon>$ $1-R^{\prime} / R$, there exists $N^{\prime}=N^{\prime}\left(R^{\prime} / R, \varepsilon, n\right)$ such that, for any sphere $S\left(x_{0}, R\right)=\left\{x \in \mathbb{R}^{n}:\left\|x-x_{0}\right\|_{2}=R\right\}$, one can find points $x_{1}, \ldots, x_{N^{\prime}}$ on that sphere such that

$$
B\left(x_{0}, 2 R-R^{\prime}\right) \backslash B\left(x_{0}, R^{\prime}\right) \subset \cup_{j=1}^{N^{\prime}} B\left(x_{j}, \varepsilon R\right)
$$

(if $R^{\prime}=0$, then we set $B\left(x_{0}, 0\right)=\emptyset$ ).

Now, take any $B=B\left(x_{0}, r\right) \in \mathscr{B}_{m}^{\Omega}$. In fact, we shall prove the aforementioned property for the "maximal" ball $B\left(x_{0}, r_{0}\right)$ with $r_{0}:=m d^{(2)}\left(x_{0}, \partial \Omega\right)$. Let $R_{j}=(j / s) r_{0}, j \in \mathbb{N}$, where $s \in \mathbb{N}$ is large enough (to be determined in the last step of the argument). Using the above geometrical fact, on each sphere $S_{j}=S\left(x_{0}, R_{j}\right), j=1,2, \ldots, s-1$, we choose finite number of points such that the balls centered at these points and with radii equal $(2 / s) r_{0}$ covering the annulus $B\left(x_{0}, R_{j+1}\right) \backslash$ $B\left(x_{0}, R_{j-1}\right)$. More precisely, given $j=1,2, \ldots, s-1$, we apply the geometrical fact with $R=R_{j}=(j / s) r_{0}$ and $R^{\prime}=R_{j-1}=$ $((j-1) / s) r_{0}$ (so that $R^{\prime} / R=((j-1) / j)$ ) and $\varepsilon=2 / j$ (so that $\left.\varepsilon>1-R^{\prime} / R\right)$. It is clear that the union of all chosen balls covers $B\left(x_{0}, r_{0}\right)$ and there is $N=\sum_{j=1}^{s-1} N^{\prime}((j-1) / j, 2 / j, n)$ of them. To verify that each of these balls is in $\mathscr{B}_{k}^{\Omega}$, take $B\left(x_{0}^{*}, r_{0}^{*}\right)$ with center lying on the sphere $S\left(x_{0}, R_{s-1}\right)$ (this is the worst case). Since, for $\omega \in \Omega^{c}$, we have $d^{(2)}\left(\omega, x_{0}\right) \leq$ $d^{(2)}\left(\omega, x_{0}^{*}\right)+d^{(2)}\left(x_{0}^{*}, x_{0}\right)$ and $d^{(2)}\left(x_{0}^{*}, x_{0}\right)=((s-1) / s) r_{0}=$ $((s-1) / s) m d^{(2)}\left(x_{0}, \partial \Omega\right)$, it is clear that

$$
d^{(2)}\left(x_{0}^{*}, \partial \Omega\right) \geq d^{(2)}\left(x_{0}, \partial \Omega\right)\left(1-m \frac{s-1}{s}\right) .
$$

Hence, if $s$ is chosen to be the least positive integer with the property $(2 / s)(1 /(1-m))<k$, then

$$
\begin{aligned}
r_{0}^{*} & =\frac{2}{s} r_{0}=\frac{2}{s} m d^{(2)}\left(x_{0}, \partial \Omega\right)<\frac{2}{s} \frac{1}{1-m} d^{(2)}\left(x_{0}^{*}, \partial \Omega\right) \\
& \leq k d^{(2)}\left(x_{0}^{*}, \partial \Omega\right)
\end{aligned}
$$

and the required property follows (note that $s$ depends on $k$ and $m$, and, hence, $N$ depends on $k, m$, and $n$, as claimed).

The results of Propositions 18 and 19 allow us to define $L_{p, \alpha}^{\mathscr{Q}}(\Omega):=L_{p, \alpha, 1 / 2}^{\mathscr{Q}}(\Omega)$ and $\|\cdot\|_{L_{p, \alpha}^{Q}(\Omega)}:=\|\cdot\|_{L_{p, \alpha, 1 / 2}^{Q}(\Omega)}$ (the choice of $k=1 / 2$ being "random") and similarly for $L_{p, \alpha}^{\mathscr{B}}(\Omega), \mathscr{L}_{p, \alpha}^{\mathscr{Q}}(\Omega), \mathscr{L}_{p, \alpha}^{\mathscr{B}}(\Omega)$, and the corresponding norms. The following theorem partially contains [24, Theorem 4.2] as a special case.

Theorem 20. Let $1 \leq p<\infty$ and $\alpha \geq-1 / p$ be given. Then, we have

$$
L_{p, \alpha}^{\mathscr{Q}}(\Omega)=L_{p, \alpha}^{\mathscr{B}}(\Omega), \quad \mathscr{L}_{p, \alpha}^{\mathscr{Q}}(\Omega)=\mathscr{L}_{p, \alpha}^{\mathscr{B}}(\Omega),
$$

with equivalence of the corresponding norms.

Proof. We focus on proving the statement concerning the Campanato spaces; the argument for the Morrey spaces is analogous (and slightly simpler). Given a cube $Q$ or a ball $B$, by $B_{Q}$ or $Q_{B}$, we will denote the ball circumscribed on $Q$ or the cube circumscribed on $B$, respectively. By the inequality $\|\cdot\|_{\infty} \leq\|\cdot\|_{2} \leq \sqrt{n}\|\cdot\|_{\infty}$, it is clear that, for $0<k \leq 1 / \sqrt{n}$, $B_{Q} \in \mathscr{B}_{\sqrt{n} k}^{\Omega}$, if $Q \in Q_{k}^{\Omega}$, and $Q_{B} \in Q_{\sqrt{n} k}^{\Omega}$, if $B \in \mathscr{B}_{k}^{\Omega}$. Moreover, $\left|B_{\mathrm{Q}}\right|=c_{1}|Q|$ and $\left|Q_{B}\right|=c_{2}|B|$, where $c_{1}$ and $c_{2}$, depend on the dimension $n$ only.

Fix $0<k<1 / \sqrt{n}$ and take $f \in \mathscr{L}_{p, \alpha, \sqrt{n} k}^{\mathscr{Q}}(\Omega)$. For any $B$ and $Q_{B}$ defined above,

$$
\begin{aligned}
& \inf _{z \in \mathbb{C}} \frac{1}{|B|^{\alpha}}\left(\frac{1}{|B|} \int_{B}|f-z|^{p}\right)^{1 / p} \\
& \quad \leq c_{2}^{\prime} \inf _{z \in \mathbb{C}} \frac{1}{\left|Q_{B}\right|^{\alpha}}\left(\frac{1}{\left|Q_{B}\right|} \int_{Q_{B}}|f-z|^{p}\right)^{1 / p} .
\end{aligned}
$$

Consequently, $\|f\|_{\mathscr{L}_{p, \alpha, k}^{\mathscr{B}}(\Omega)} \leq c_{2}^{\prime}\|f\|_{\mathscr{L}_{p, \alpha, \sqrt{d k}}^{\mathscr{Q}}(\Omega)}$ which also shows that $\mathscr{L}_{p, \alpha, \sqrt{n} k}^{\mathscr{Q}}(\Omega) \subset \mathscr{L}_{p, \alpha, k}^{\mathscr{B}}(\Omega)$. The results of Propositions 18 and 19 now give $\mathscr{L}_{p, \alpha}^{\mathscr{Q}}(\Omega) \subset \mathscr{L}_{p, \alpha}^{\mathscr{B}}(\Omega)$ and $\|\cdot\|_{\mathscr{L}_{p, \alpha}^{\mathscr{B}}(\Omega)} \leq$ $C\|\cdot\|_{\mathscr{L}_{p, \alpha}^{Q}(\Omega)}$. The opposite inclusion and inequality are proved in an analogous way.

Clearly, the concept of Morrey and Campanato spaces on open proper subsets of $\mathbb{R}^{n}$ may be generalized to open proper subsets of a general quasimetric space $X$. See [4, Section 5], where the concept of local maximal operators in such framework was mentioned. Finally, we mention that the presented concept of locality for open proper subdomains in the Euclidean spaces is rather common. See, for instance, the recent paper [25] where the regularity of the local HardyLittlewood operator was studied and the paper [12] where notions of local fractional operators were introduced and studied, in both cases in the setting of $\Omega \subset \mathbb{R}^{n}$ with the locality function $\Omega \ni x \mapsto \operatorname{dist}\left(x, \mathbb{R}^{n} \backslash \Omega\right)$. 


\section{Boundedness of Operators on Local Morrey Spaces}

Boundedness of classical operators of harmonic analysis on Morrey spaces was investigated in a vast number of papers; see, for instance, $[3,5,17,26-30]$ and references cited there.

In this section, we assume the system $(X, d, \mu, \rho)$ to be fixed. We begin with a result on the boundedness of local Hardy-Littlewood maximal operator between local Morrey spaces. For the notational convenience, let $\tau_{K}=K(2 K+1)$, where $K$ is the constant from the quasitriangle inequality (if $d$ is a metric, then $K=1$ and $\tau_{K}=3$ ). Observe that the assumptions we impose in Proposition 21 are satisfied, for instance, when $\mu(B) \simeq r(B)^{n}$, for some $n>0$, uniformly in $B \in \mathscr{B}_{\rho}, \varphi(r)=r^{\alpha},-n / p \leq \alpha \leq 0$, and $\mu$ satisfies the $\rho$-local $\tau_{K}$-condition. Recall also that when it comes to the boundedness of $M_{1,1, \rho}$ on $L^{p}(X, \mu)$, we have the conclusion of Proposition 4 to our disposal.

Proposition 21. Let $1 \leq p<\infty$ and $\varphi:(0, \infty) \rightarrow(0, \infty)$ be a nonincreasing function such that $\phi(B)=\varphi(r(B))$ satisfies

$$
\phi\left(\tau_{K} B\right) \mu\left(\tau_{K} B\right)^{1 / p} \lesssim \phi(B) \mu(B)^{1 / p}
$$

uniformly in $B \in \mathscr{B}_{\rho}$. If $M_{1,1, \rho}$ is bounded on $L^{p}(X, \mu)$, then, it is also bounded from $L_{p, \phi, \tau_{K} \rho}(X)$ to $L_{p, \phi, \rho}(X)$.

Proof. For the notational convention, let $M_{\rho}:=M_{1,1, \rho}$; that is,

$$
M_{\rho} f(x)=\sup _{x \in B \in \mathscr{B}_{\rho}} \frac{1}{\mu(B)} \int_{B}|f| d \mu .
$$

Take $f \in L_{p, \phi, \tau_{K} \rho}(X)$, fix a ball $B \in \mathscr{B}_{\rho}$, and consider the splitting $f=f_{1}+f_{2}$ adjusted to $B$ in the sense that $f_{1}=f \chi_{\tau_{K} B}$. Then, for any $x \in B$,

$$
M_{\rho} f_{2}(x) \leq \sup _{B^{\prime}} \frac{1}{\mu\left(B^{\prime}\right)} \int_{B^{\prime}}|f| d \mu,
$$

where the supremum is taken over all balls $B^{\prime} \in \mathscr{B}_{\rho}$ such that $x \in B^{\prime}$ and $r\left(B^{\prime}\right)>r(B)$. If $x \in B$ and $B^{\prime}$ is one of such balls, then the fact that $\varphi$ is nonincreasing gives

$$
\begin{aligned}
\frac{1}{\phi(B)} \frac{1}{\mu\left(B^{\prime}\right)} \int_{B^{\prime}}|f| d \mu & \leq \frac{1}{\varphi(r(B))}\left(\frac{1}{\mu\left(B^{\prime}\right)} \int_{B^{\prime}}|f|^{p} d \mu\right)^{1 / p} \\
& \leq \frac{1}{\phi\left(B^{\prime}\right)}\left(\frac{1}{\mu\left(B^{\prime}\right)} \int_{B^{\prime}}|f|^{p} d \mu\right)^{1 / p} \\
& \leq\|f\|_{L_{p, \phi, \rho}}
\end{aligned}
$$

Consequently,

$$
\frac{1}{\phi(B)} M_{\rho} f_{2}(x) \leq\|f\|_{L_{\rho, \phi, \rho}}, \quad x \in B
$$

This estimate, subadditivity of $M_{\rho}$, and the assumption that $M_{\rho}$ is bounded on $L^{p}(X, \mu)$ give

$$
\begin{aligned}
& \frac{1}{\phi(B)}\left(\frac{1}{\mu(B)} \int_{B}\left(M_{\rho} f\right)^{p} d \mu\right)^{1 / p} \\
& \quad \leq \sum_{i=1,2} \frac{1}{\phi(B)}\left(\frac{1}{\mu(B)} \int_{B}\left(M_{\rho} f_{i}\right)^{p} d \mu\right)^{1 / p} \\
& \quad \leq c_{p} \frac{1}{\phi(B) \mu(B)^{1 / p}}\left(\int_{\tau_{K} B}|f|^{p} d \mu\right)^{1 / p}+\|f\|_{L_{p, \phi, \rho}} \\
& \quad \leq c_{p}^{\prime} \frac{1}{\phi\left(\tau_{K} B\right) \mu\left(\tau_{K} B\right)^{1 / p}}\left(\int_{\tau_{K} B}|f|^{p} d \mu\right)^{1 / p}+\|f\|_{L_{p, \phi, \tau_{K} \rho}} \\
& \leq c_{p}^{\prime \prime}\|f\|_{L_{p, \phi, \tau} \rho} .
\end{aligned}
$$

This shows the required estimate $\left\|M_{\rho} f\right\|_{L_{p, \phi, \rho}} \lesssim\|f\|_{L_{p, \phi, \tau} \rho}$.

Remark 22. Consider the global case, $\rho \equiv \infty$. To rediscover the classical result of Chiarenza and Frasca, [31, Theorem 1], which is the boundedness of the usual Hardy-Littlewood operator on the space $L_{p, \lambda}\left(\mathbb{R}^{n}\right), 1<p<\infty$ and $0<\lambda<n$ (see Remark 7), take $\varphi(r)=r^{(\lambda-n) / p}$ which is decreasing. The assumption (92), with $\mu$ being Lebesgue measure and the metric being $d^{(\infty)}$ is obviously satisfied (clearly the usual Hardy-Littlewood operator is also bounded on $L^{p}\left(\mathbb{R}^{n}\right)$ ).

Similarly, if $(X, d, \mu)$ is a space of homogeneous type and $\varphi(r)=r^{\alpha}, \alpha \leq 0$, so that $\phi=\phi_{r, \alpha}$, then condition (92) is satisfied and, hence, $M_{\mu}=M_{1,1}$, the Hardy-Littlewood operator associated to $\mu$, maps boundedly $L_{p, \phi_{r, \alpha}}$ into itself.

In the literature, several variants of fractional integrals over quasimetric measure spaces are considered. Here, we shall consider a variant in the setting of a quasimetric measure spaces $(X, d, \mu)$ with $\mu$ satisfying the upper growth condition (23) with $\tau=n$. For any appropriate function $f$ and $0<\beta<$ $n$, we define the fractional integral operator $I_{\beta}$ by letting

$$
I_{\beta} f(x)=\int_{X} \frac{f(y)}{d(x, y)^{n-\beta}} d \mu(y), \quad x \in X .
$$

For functions $\varphi, \psi:(0, \infty) \rightarrow(0, \infty)$, we shall consider the following conditions (compare them with the assumptions imposed in [32]):

$$
\begin{gathered}
\frac{\varphi(r)}{r} \text { is nonincreasing; } \\
\int_{r}^{\infty} t^{\beta-1} \varphi(t) d t \lesssim \psi(r) \quad r>0 .
\end{gathered}
$$

Proposition 23. Let $1 \leq p, q<\infty$ and $\varphi, \psi:(0, \infty) \rightarrow$ $(0, \infty)$ be functions satisfying (99). In addition, assume that $\phi(B)=\varphi(r(B))$ and $\Psi(B)=\psi(r(B))$ satisfy

$$
\phi\left(\tau_{K} B\right) \mu\left(\tau_{K} B\right)^{1 / p} \lesssim \Psi(B) \mu(B)^{1 / q},
$$


uniformly in $B \in \widetilde{\mathscr{B}}$. If $I_{\beta}$ is bounded from $L^{p}(X, \mu)$ to $L^{q}(X, \mu)$, then, for any $\rho$, it is also bounded from $L_{p, \phi}(X)$ to $L_{q, \Psi, \rho}(X)$.

Proof. Since $\|\cdot\|_{L_{q, \Psi, \rho}} \leq\|\cdot\|_{L_{q, \Psi}}$, it is sufficient to consider the case $\rho \equiv \infty$. The estimate to be proved is

$$
\frac{1}{\Psi(B)}\left(\frac{1}{\mu(B)} \int_{B}\left|I_{\beta} f\right|^{q} d \mu\right)^{1 / q} \lesssim\|f\|_{L_{p, \phi}},
$$

uniformly in $B \in \widetilde{\mathscr{B}}$ and $f \in L_{p, \phi}(X)$. Take $B=B(a, r)$ and $f \in L_{p, \phi}(X)$ and consider the decomposition $f=f_{1}+f_{2}$, $f_{1}=f \chi_{\tau_{K} B}$, and $\tau_{K}:=2 K$. It suffices to verify (101) with $f$ replaced by $f_{i}, i=1,2$, on the left hand side of this estimate. For $f_{1}$, using the assumption on the $L^{p}-L^{q}$ boundedness of $I_{\beta}$ and (100), we write

$$
\begin{aligned}
& \frac{1}{\Psi(B)}\left(\frac{1}{\mu(B)} \int_{B}\left|I_{\beta} f_{1}\right|^{q} d \mu\right)^{1 / q} \\
& \quad \leq \frac{1}{\Psi(B) \mu(B)^{1 / q}}\left\|I_{\beta} f_{1}\right\|_{L^{q}(\mu)} \\
& \quad \lesssim \frac{1}{\Psi(B) \mu(B)^{1 / q}}\left\|f_{1}\right\|_{L^{p}(\mu)} \\
& \quad \leq \frac{1}{\phi\left(\tau_{K} B\right)}\left(\frac{1}{\mu\left(\tau_{K} B\right)} \int_{\tau_{K} B}|f|^{p} d \mu\right)^{1 / p} \\
& \quad \leq\|f\|_{L_{p, \phi}}
\end{aligned}
$$

For $f_{2}$, note that, for any $x \in B$, we have $B\left(a, \tau_{K} r\right)^{c} \subset B(x, r)^{c}$ ( $A^{c}$ denotes the completion of $A$ in $X$ ), and, therefore,

$$
\begin{aligned}
\left|I_{\beta} f_{2}(x)\right| & \leq \int_{\left(\tau_{K} B\right)^{c}} \frac{|f(y)|}{d(x, y)^{n-\beta}} d \mu(y) \\
& \leq \int_{B(x, r)^{c}} \frac{|f(y)|}{d(x, y)^{n-\beta}} d \mu(y) \\
& =\sum_{j=0}^{\infty} \int_{\left\{y: 2^{j} r \leq d(x, y)<2^{j+1} r\right\}} \frac{|f(y)|}{d(x, y)^{n-\beta}} d \mu(y) \\
& \leq \sum_{j=0}^{\infty}\left(2^{j} r\right)^{\beta-n} \int_{\left\{y: 2^{j} r \leq d(x, y)<2^{j+1} r\right\}}|f(y)| d \mu(y) \\
& \leq \sum_{j=0}^{\infty}\left(2^{j} r\right)^{\beta} \frac{1}{\mu\left(B\left(x, 2^{j+1} r\right)\right)} \int_{B\left(x, 2^{j+1} r\right)}|f(y)| d \mu(y) \\
& \leq\left(\sum_{j=0}^{\infty}\left(2^{j} r\right)^{\beta} \phi\left(B\left(x, 2^{j+1} r\right)\right)\right)\|f\|_{L_{p, \phi}} \\
& \leq \int_{r}^{\infty} t^{\beta-1} \varphi(t) d t\|f\|_{L_{p, \phi}} \\
& \left.\leqslant(r)\|f\|_{L_{p, \phi} \cdot} .103\right)
\end{aligned}
$$

With this pointwise estimate, it follows that

$$
\frac{1}{\Psi(B(a, r))}\left(\frac{1}{\mu(B(a, r))} \int_{B(a, r)}\left|I_{\beta} f_{2}\right|^{q} d \mu\right)^{1 / q} \leqslant\|f\|_{L_{p, \phi}} .
$$

The proof is complete.

Remark 24. García-Cuerva and Gatto proved that [33, Corollary 3.3], for a metric measure space $(X, d, \mu)$, satisfying (23), $I_{\beta}$ is bounded from $L^{p}(\mu)$ to $L^{q}(\mu)$ provided that $1<p<n / \beta$ and $1 / q=1 / p-\beta / n$. The assumption that $d$ is a metric may be relaxed; see [1], and in fact we can assume $(X, d, \mu)$ to be a quasi-metric measure space satisfying (23). Let $\alpha<-\beta$. Then, $\varphi(r):=r^{\alpha}$ and $\psi(r):=r^{\alpha+\beta}$ satisfy (99). In addition, if we assume that $\mu$ satisfies $\mu(B(x, r)) \simeq r^{n}$, uniformly in $x \in X$ and $r>0$, then (100) holds with constraints on $p, q, \beta$ as above. Therefore, with all these assumptions, $I_{\beta}$ is bounded from $L_{p, \phi}(X)$ to $L_{q, \Psi}(X)$.

\section{Conflict of Interests}

The authors declare that there is no conflict of interests regarding the publication of this paper.

\section{Acknowledgments}

The research was initiated when Krzysztof Stempak visited the Department of Mathematics of Zhejiang University of Science and Technology, China, in April 2013. He is thankful for the warm hospitality he received. The authors would like to thank the referees for their careful comments. The research of Krzysztof Stempak is supported by NCN of Poland under Grant 2013/09/B/ST1/02057. The research of Xiangxing Tao is supported by NNSF of China under Grants nos. 11171306 and 11071065.

\section{References}

[1] K. Stempak, "On quasi-metric measure spaces," preprint. In press.

[2] E. Nakai, “The Campanato, Morrey and Hölder spaces on spaces of homogeneous type," Studia Mathematica, vol. 176, no. 1, pp. $1-19,2006$.

[3] Y. Sawano and H. Tanaka, "Morrey spaces for non-doubling measures," Acta Mathematica Sinica. English Series, vol. 21, no. 6, pp. 1535-1544, 2005.

[4] C.-C. Lin, K. Stempak, and Y.-S. Wang, "Local maximal operators on measure metric spaces," Publicacions Matemàtiques, vol. 57, no. 1, pp. 239-264, 2013.

[5] D. Yang, D. Yang, and Y. Zhou, "Localized Morrey-Campanato spaces on metric measure spaces and applications to Schrödinger operators," Nagoya Mathematical Journal, vol. 198, pp. 77-119, 2010

[6] L. Liu, Y. Sawano, and D. Yang, "Morrey-type spaces on Gauss measure spaces and boundedness of singular integrals," Journal of Geometric Analysis, vol. 24, no. 2, pp. 1007-1051, 2014.

[7] T. Hytönen, "A framework for non-homogeneous analysis on metric spaces, and the RBMO space of Tolsa," Publicacions Matemàtiques, vol. 54, no. 2, pp. 485-504, 2010. 
[8] X. Tolsa, "BMO, $H^{1}$, and Calderón-Zygmund operators for non doubling measures," Mathematische Annalen, vol. 319, no. 1, pp. 89-149, 2001.

[9] F. Nazarov, S. Treil, and A. Volberg, "Weak type estimates and Cotlar inequalities for Calderón-Zygmund operators on nonhomogeneous spaces," International Mathematics Research Notices, no. 9, pp. 463-487, 1998.

[10] W. Chen and E. Sawyer, "A note on commutators of fractional integrals with $R B M O(\mu)$ functions," Illinois Journal of Mathematics, vol. 46, no. 4, pp. 1287-1298, 2002.

[11] T. Heikkinen, J. Lehrbäck, J. Nuutinen, and H. Tuominen, "Fractional maximal functions in metric measure spaces," Analysis and Geometry in Metric Spaces, vol. 1, pp. 147-162, 2012.

[12] T. Heikkinen, J. Kinnunen, J. Korvenpää, and H. Tuominen, "Regularity of the local fractional maximal function," http:// arxiv.org/abs/1310.4298.

[13] J. Heinonen, Lectures on Analysis on Metric Spaces, Springer, New York, NY, USA, 2001.

[14] Y. Sawano, "Sharp estimates of the modified Hardy-Littlewood maximal operator on the nonhomogeneous space via covering lemmas," Hokkaido Mathematical Journal, vol. 34, no. 2, pp. 435-458, 2005.

[15] Y. Terasawa, "Outer measures and weak type $(1,1)$ estimates of Hardy-Littlewood maximal operators," Journal of Inequalities and Applications, vol. 2006, Article ID 15063, 13 pages, 2006.

[16] J. Petree, "On the theory of $\mathscr{L}_{p, \lambda}$ spaces," Journal of Functional Analysis, vol. 4, pp. 71-87, 1969.

[17] Y. Sawano, "Generalized Morrey spaces for non-doubling measures," Nonlinear Differential Equations and Applications, vol. 15, no. 4-5, pp. 413-425, 2008.

[18] E. Nakai, "A characterization of pointwise multipliers on the Morrey spaces," Scientiae Mathematicae, vol. 3, no. 3, pp. 445454, 2000.

[19] J. Mateu, P. Mattila, A. Nicolau, and J. Orobitg, "BMO for nondoubling measures," Duke Mathematical Journal, vol. 102, no. 3, pp. 533-565, 2000.

[20] P. W. Jones, "Extension theorems for BMO," Indiana University Mathematics Journal, vol. 29, no. 1, pp. 41-66, 1980.

[21] C. T. Zorko, "Morrey space," Proceedings of the American Mathematical Society, vol. 98, no. 4, pp. 586-592, 1986.

[22] C. B. Morrey, Jr., "On the solutions of quasi-linear elliptic partial differential equations," Transactions of the American Mathematical Society, vol. 43, no. 1, pp. 126-166, 1938.

[23] S. Campanato, "Proprietà di una famiglia di spazi funzionali," Annali della Scuola Normale Superiore di Pisa, vol. 18, pp. 137160, 1964.

[24] C.-C. Lin and K. Stempak, "Atomic $H^{p}$ spaces and their duals on open subsets of $\mathbb{R}^{n}$," Forum Mathematicum, 2013.

[25] H. Luiro, "On the regularity of the Hardy-Littlewood maximal operator on subdomains of $R^{n}$," Proceedings of the Edinburgh Mathematical Society, vol. 53, no. 1, pp. 211-237, 2010.

[26] Eridani, H. Gunawan, E. Nakai, and Y. Sawano, "Characterizations for the generalized fractional integral operators on Morrey spaces," Mathematical Inequalities \& Applications. In press.

[27] Y. Sawano, S. Sugano, and H. Tanaka, "Generalized fractional integral operators and fractional maximal operators in the framework of Morrey spaces," Transactions of the American Mathematical Society, vol. 363, no. 12, pp. 6481-6503, 2011.

[28] W. Yuan, W. Sickel, and D. Yang, Morrey and Campanato Meet Besov, Lizorkin and Triebel, vol. 2005 of Lecture Notes in Mathematics, Springer, Berlin, Germany, 2010.
[29] I. Sihwaningrum and Y. Sawano, "Weak and strong type estimates for fractional integral operators on Morrey spaces over metric measure spaces," Eurasian Mathematical Journal, vol. 4, no. 1, pp. 76-81, 2013.

[30] Y. Shi and X. Tao, "Some multi-sublinear operators on generalized Morrey spaces with non-doubling measures," Journal of the Korean Mathematical Society, vol. 49, no. 5, pp. 907-925, 2012.

[31] F. Chiarenza and M. Frasca, "Morrey spaces and HardyLittlewood maximal function," Rendiconti di Matematica e delle sue Applicazioni. Serie VII, vol. 7, no. 3-4, pp. 273-279, 1987.

[32] E. Nakai, "Hardy-Littlewood maximal operator, singular integral operators and the Riesz potentials on generalized Morrey spaces," Mathematische Nachrichten, vol. 166, pp. 95-103, 1994.

[33] J. García-Cuerva and A. E. Gatto, "Boundedness properties of fractional integral operators associated to non-doubling measures," Studia Mathematica, vol. 162, no. 3, pp. 245-261, 2004 . 


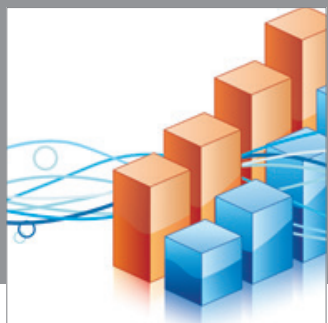

Advances in

Operations Research

mansans

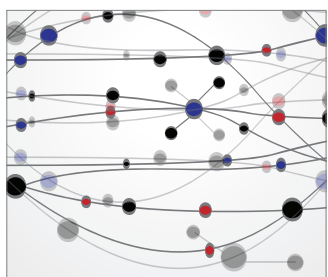

The Scientific World Journal
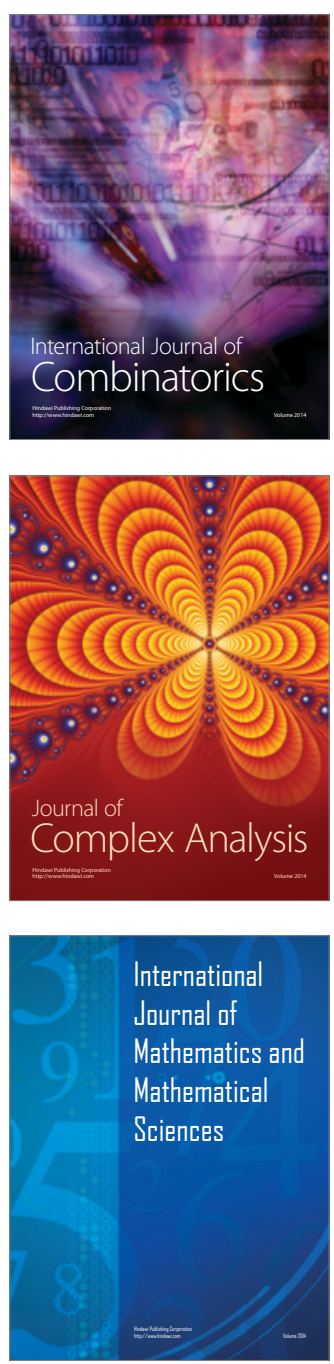
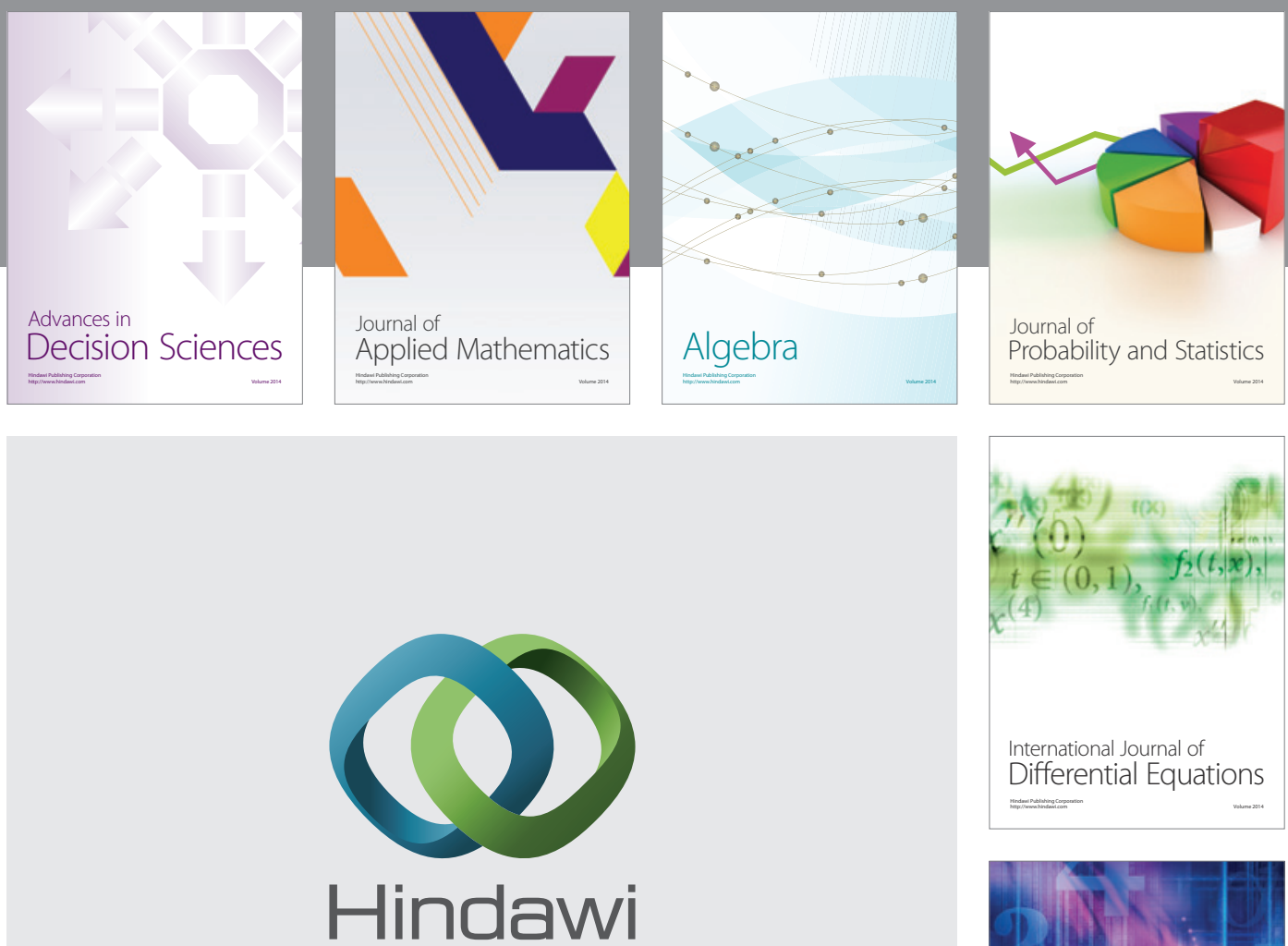

Submit your manuscripts at http://www.hindawi.com
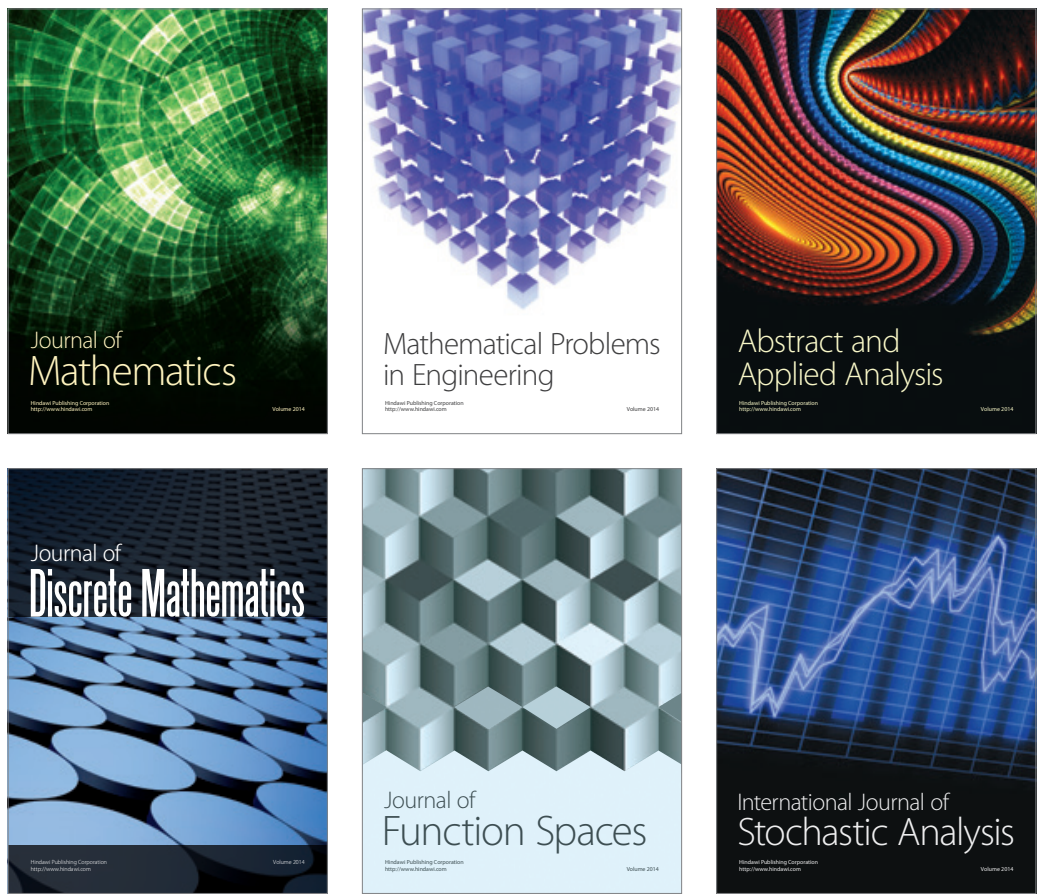

Journal of

Function Spaces

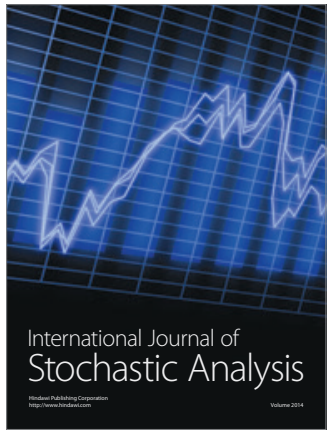

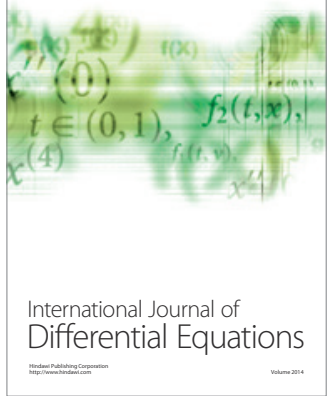
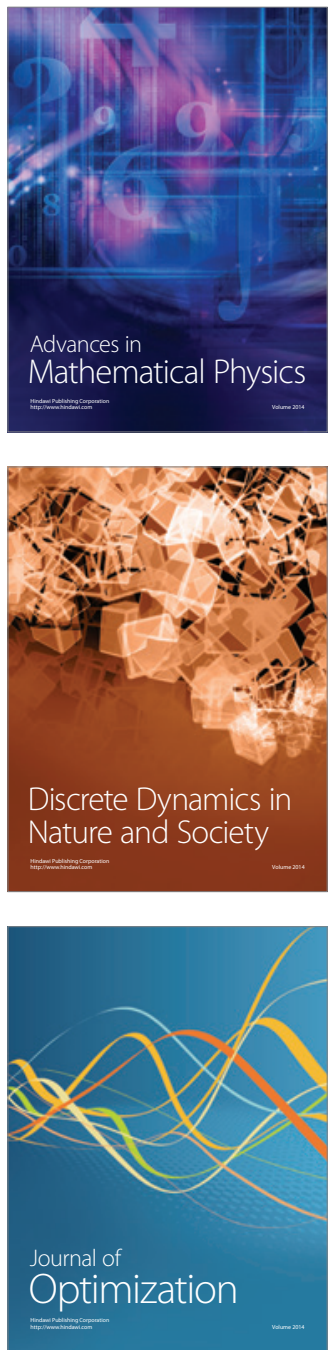